\title{
Lysine Acetylation: Elucidating the Components of an Emerging Global Signaling Pathway in Trypanosomes
}

\author{
Victoria Lucia Alonso $^{1,2}$ and Esteban Carlos Serra ${ }^{1,2}$ \\ ${ }^{1}$ Departamento de Microbiología, Facultad de Ciencias Bioquímicas y Farmacéuticas, Universidad Nacional de Rosario, \\ Suipacha 531, Rosario 2000, Argentina \\ ${ }^{2}$ Instituto de Biología Molecular y Celular de Rosario, CONICET-UNR, Suipacha 590, Rosario 2000, Argentina
}

Correspondence should be addressed to Esteban Carlos Serra, eserra@fbioyf.unr.edu.ar

Received 17 April 2012; Revised 20 July 2012; Accepted 30 July 2012

Academic Editor: Andrea Silvana Rópolo

Copyright ( $) 2012$ V. L. Alonso and E. C. Serra. This is an open access article distributed under the Creative Commons Attribution License, which permits unrestricted use, distribution, and reproduction in any medium, provided the original work is properly cited.

In the past ten years the number of acetylated proteins reported in literature grew exponentially. Several authors have proposed that acetylation might be a key component in most eukaryotic signaling pathways, as important as phosphorylation. The enzymes involved in this process are starting to emerge; acetyltransferases and deacetylases are found inside and outside the nuclear compartment and have different regulatory functions. In trypanosomatids several of these enzymes have been described and are postulated to be novel antiparasitic targets for the rational design of drugs. In this paper we overview the most important known acetylated proteins and the advances made in the identification of new acetylated proteins using high-resolution mass spectrometry. Also, we summarize what is known so far about the acetyltransferases and deacetylases in eukaryotes, focusing on trypanosomes and their potential use as chemotherapeutic targets.

\section{Introduction}

Lysine $(\mathrm{K})$ acetylation is a reversible and highly regulated posttranslational modification (PTM) involved in a wide range of cellular processes in eukaryotes. It has been proposed that acetylation might have the same relevance as phosphorylation, although no acetylation cascade has been reported to date [1]. During the past four decades lysine acetylation was associated with important roles in the regulation of nuclear transcription. Recently, proteomewide analyses revealed a large number of acetylated proteins in the cytoplasm and the mitochondria, including most of the enzymes involved in the intermediate metabolism. These findings suggest a central role for an acetylationrelated regulatory mechanism in cell development inside and outside the nucleus [2].

Lysine acetylation is catalyzed by acetyltransferases, which transfer the acetyl group of acetyl CoA to the $\varepsilon$-amino group of an internal lysine residue. The reverse reaction is catalyzed by deacetylases. Both types of enzymes are usually part of protein complexes and were named histone acetyltransferases (HATs) and histone deacetylases (HDACs) because they were initially found to target histones. Later on they were shown to target nonhistone proteins as well. Allis et al. proposed in 2007 that these names should be changed to lysine acetyltransferases (KATs) and lysine deacetylases (KDACs), names that better correlate with the broader substrate specificity of these enzymes [3].

Trypanosoma cruzi, Trypanosoma brucei, and Leishmania spp. (named collectively Tritryps) are trypanosomatid parasites (order Kinetoplastida) that cause millions of deaths in tropical and subtropical regions of the world [4]. Trypanosomatids branched early from the eukaryotic lineage, and this is reflected in some unique characteristics like polycistronic transcription, transsplicing, and the involvement of RNA polymerase $I$ in the transcription of certain protein coding genes [5]. They exhibit complex life cycles, with different developmental stages alternating between vertebrate and invertebrate hosts [6-8].

Trypanosoma brucei is transmitted to humans through the bite of tse-tse flies (Glossina spp.) and is responsible for african sleeping sickness. In mammals, the parasite survives 
free in the bloodstream and is able to evade the host immune response through antigenic variation [7]. Trypanosoma cruzi is transmitted through hematophagous insect vectors of the Reduvidae and Hemiptera families and is the causative agent of Chagas disease. Once in the mammalian host, it multiplies intracellularly (amastigote stage) and differentiates into the nonreplicative infective form present in the bloodstream (trypomastigote stage) [9]. Leishmania spp. is transmitted by the bite of sand flies (Phlebotomies) and has two main life cycle morphologies: the intracellular amastigote in the mammalian host and the promastigote in the fly. Clinical symptoms of leishmaniasis vary from cutaneous and mucocutaneous to visceral depending on the species [8].

The aim of this paper is to describe the general bases of lysine acetylation as well as the enzymes responsible for this modification, focusing on trypanosomatids. Additionally, we describe the possible use of lysine acetyltransferases and deacetylases as chemotherapeutic targets for parasitic diseases.

\section{Acetylation: A General Posttranslational Modification}

2.1. Acetylation of Histone and Nonhistone Proteins. Lysine acetylation was initially discovered as a posttranslational modification of histones in the 1960s [10,11]. Histone Nterminal tails are extended from the core of the nucleosomes and are modified by acetylation and other posttranslational modifications such as phosphorylation, methylation, ubiquitination, and sumoylation. These PTMs can alter DNA-histone interactions or the binding of other proteins, such as transcription factors to chromatin. It has been suggested that distinct modifications present on histone tails may act sequentially or in combination to form a code. This "histone code" is read by other proteins or protein modules and determines a variety of nuclear events [12]. Histone acetylation occurs on the $\mathcal{\varepsilon}$-amino groups of specific lysine residues and has emerged as a modification of major regulatory significance. This chromatin-based mechanism modulates the accessibility of genetic information during transcription, DNA replication, recombination, and repair [13]. The addition of an acetyl group neutralizes the positive charge and changes the overall size of the modified amino acid along with the local hydrophobicity of the protein. These variations have a significant impact on the conformation and function (e.g., its enzymatic activity) of the polypeptide. Acetylation on lysine residues can also generate docking sites for the binding of proteins, a topic that will be discussed later in this paper [14].

Chromatin in trypanosomatids is less condensed than in most eukaryotic cells and appears to be organized in $10 \mathrm{~nm}$ filaments [24-26]. These organisms have several copies of histones genes ( $\mathrm{H} 1, \mathrm{H} 2 \mathrm{~A}, \mathrm{H} 2 \mathrm{~B}, \mathrm{H} 3$, and $\mathrm{H} 4)$. However, they are extremely divergent from those found in other eukaryotes [27]. Numerous histone PTMs were reported in trypanosomatids. In general terms, T. cruzi histones $\mathrm{H} 4$ and $\mathrm{H} 2 \mathrm{~A}$ are mainly acetylated, whereas histones $\mathrm{H} 3$ and $\mathrm{H} 2 \mathrm{~B}$ are methylated. Histone $\mathrm{H} 4$ modifications have been extensively studied in T. cruzi, lysine residues $4,10,14$, and 54 are acetylated, $\mathrm{K} 18$ is monomethylated and arginine at position 53 is dimethylated [28]. In T. brucei, histones H4 K2 and K5 are also acetylated $[29,30]$. The Trytrips genomes contain coding sequences for methyltransferases, acetyltransferases and deacetylases that might be responsible for the modifications of histone N-terminal domains in these organisms [31]. Apparently, in trypanosomatids different functional states of chromatin correlate with specific posttranslational histone modifications and histone variants. It has been demonstrated that there is a direct correlation between certain PTMs and transcriptional initiation/termination, cell cycle progression, and telomeric silencing [32].

Several authors went further with the characterization of histones PTMs and described in some detail the implications of acetylation on trypanosomatids histones. In T. cruzi, histone $\mathrm{H} 4$ acetylated at lysine $4(\mathrm{H} 4 \mathrm{~K} 4 \mathrm{ac})$ was detected in densely packed chromatin, whereas acetylation at lysines 10 and 14 (H4K10ac, H4K14ac) was present in less condensed zones. Acetylation at K10 and K14 of histone H4 increases during the rupture of the DNA double strand, which suggests an important role for this PTM in chromatin organization. In addition, $\mathrm{H} 4 \mathrm{~K} 4 \mathrm{ac}$ levels decrease in the nonreplicative forms of the parasite (trypomastigotes), whereas H4K10ac and H4K14ac levels remain unchanged. This indicates that K4 might be involved in the synthesis of new chromatin during replication [33]. Although trypanosomatids histones are quite divergent from their eukaryotic counterparts, mainly in the regions that are subjected to PTMs, epigenetic regulation seems to be very important in these organisms. In fact, since Tritryps lack canonical promoter-driven transcriptional regulation, epigenetic regulation is proposed to be the major mechanisms for global transcriptional control in these organisms.

To examine the genome-wide distribution of chromatin components, chromatin immunoprecipitation and sequencing (ChIP-seq) experiments were performed in T. brucei and L. mayor. Siegel et al. showed that in T. brucei H4K10ac is enriched at polymerase II transcription start sites. The authors also reported that histone variants H2AZ and $\mathrm{H} 2 \mathrm{BV}$ might be associated with the transcription process [37]. Thomas et al. showed that protein-coding genes in L. major contain acetylated histone $\mathrm{H} 3$ at the origins of polycistronic transcription [38]. Thus, acetylation of histone tails correlates with an increment in transcription rates. PTMs and the presence of canonical histone variants might act as chromatin signals indicating transcription start and termination points in trypanosomatids. These findings suggest that histone modifications play crucial roles in transcription initiation and termination in trypanosomes and that destabilization of nucleosomes by histone variants is an ancient and general mechanism of transcription initiation.

Acetylation of other nuclear proteins such as the High Mobility Group (HMG) proteins was reported in the past [39]. In T. cruzi, HMG proteins were described [40] and a High Mobility Group B (TcHMGB) family member was recently characterized [41]. TcHMGB has orthologues in T. brucei and Leishmania; all of them display two HMG box domains and lack the acidic C-terminal tail characteristic 
TABLE 1: Acetylomes from different organisms.

\begin{tabular}{|c|c|c|c|}
\hline & Number of acetylation sites & Number of acetylated proteins & Reference \\
\hline $\begin{array}{l}\text { HeLa cells and mouse liver } \\
\text { mitochondria }\end{array}$ & 388 & $\begin{array}{l}195 \text { (37 from the cytosolic fraction, } 38 \text { from the } \\
\text { nuclear fraction and } 133 \text { from mitochondria) }\end{array}$ & Kim et al., 2006 [15] \\
\hline E. coli & 125 & 84 & Yu et al., 2008 [16] \\
\hline E. coli & 138 & 91 & Zhang et al., 2009 [17] \\
\hline $\begin{array}{l}\text { MV4-11 cells, A549 cells } \\
\text { and Jurkat cells }\end{array}$ & more than 3600 & 1750 & Choudhary et al., 2009 [18] \\
\hline Human liver & 1300 & 1047 & Zhao et al., 2010 [19] \\
\hline S. enterica & 235 & 191 & Wang et al., 2010 [20] \\
\hline D. melanogaster & 1981 & & Weinert et al., 2011 [21] \\
\hline A. thaliana & 91 & 74 & Finkemeier et al., 2011 [22] \\
\hline T. gondii & 411 & 274 & Jeffers and Sullivan Jr., 2012 [23] \\
\hline
\end{tabular}

of mammalian HMGBs. Instead, Tritryps HMGBs display a unique $\mathrm{N}$-terminal tail of 110 amino acids. In other species, the acetylation status of HMGB proteins plays a crucial role since it can alter their function and location. Also, it seems to be a signal for extranuclear localization of the proteins as well [42-45]. TcHMGB has numerous lysine residues that are predicted to be acetylated, and seven of them are present in the predicted nuclear localization signal (NLS) on its aminoterminal domain [41].

Alpha-tubulin was the first nonnuclear protein described to be acetylated; however, the function of this PTM is not completely understood yet. In 1985, L'Hernault and Rosenbaum reported that chlamydomonas axonemal microtubules contained acetylated $\alpha$-tubulin. These authors proposed that reversible tubulin acetylation might control the assembly and disassembly of axonemal microtubules [52-54]. Microtubules (MTs) are dynamic cytoskeletal filaments composed of $\alpha / \beta$ tubulin heterodimers. These structures play key roles in diverse cellular functions including structural support, organelle localization, chromosome segregation, and intracellular trafficking. Besides acetylation, microtubules may undergo other posttranslational modifications, which are proposed to generate a code similar to the histone code. It is thought that microtubule-associated proteins might read this code and regulate the MTs structure [55]. Initially, it was thought that MT stabilization was a consequence of MT acetylation [56, 57]. Later, several authors demonstrated that acetylation of MTs does not necessarily affect their stability $[58,59]$.

In trypanosomatids, where several tubulin PTMs have been described, acetylation of $\alpha$-tubulin at lysine 40 is particularly well characterized. In Tritryps acetylated $\alpha$ tubulin is found in the subpellicular microtubules that run along the inner surface of the parasites. This array of MT plays an essential role in the maintenance of the parasites shape and is remodelled during the differentiation process throughout its life cycle [60-62]. This PTM is also present in the axoneme of the flagellum and in the ephemeral microtubules of the mitotic spindle of T. brucei [63]. The presence of acetylated tubulin in early mitotic spindles in several organisms and the fact that protozoa like T. brucei and T. cruzi exhibits global $\alpha$-tubulin acetylation reinforces the idea that this PTM is not restricted to stable MTs.

2.2. Acetylation versus Phosphorylation. Lysine acetylation is found in all domains of life from bacteria to humans, but small progress has been made towards understanding the exact biological role of this modification. The data provided by proteome-wide analyses helps to generate new hypotheses that will increase the current understanding of lysine acetylation in a biological context. In the last ten years, several authors using high-resolution mass spectrometry identified thousands of acetylated proteins in different organisms. The proteins identified are involved in: transcription, DNA repair, chromatin remodeling, cell cycle, splicing, metabolism, cytoskeletal dynamics, apoptosis, nuclear import, protein folding, and cellular signaling among others $[15-22,64,65]$ (Table 1). All this evidence suggest that the size of the "lysine acetylome" is comparable to that of the "phosphoproteome" [1, 2, 15-22, 64, 65]. Kouzarides predicted in 2000 that acetylation might rival phosphorylation as a regulator of cellular processes [1]. Even though evidence clearly shows that acetylation is widely spread in all domains of life, the philosophy of acetylation clearly differs to that of phosphorylation. In contrast to what happens with phosphorylation, an acetylation cascades do not exist. Some acetyltransferases and deacetylases were described to be acetylated themselves; however, no acetylating enzymes able to catalyze the transfer of acetyl groups from one protein to another have been described and they are not supposed to exist either. Another interesting fact is that the number of acetyltransferases and deacetylases in eukaryotes is significantly lower than that of kinases and phosphatases. These observations suggest that the acetylation-dependant regulation is much more global than phosphorylation.

Currently there is an increasing interest for acetylation in protozoa, even though information about this topic is still scattered. Until now, the most studied acetylated proteins in these organisms were histones and tubulin; however, the first proteome-wide analysis of acetylated proteins in a protozoan organism was published this year. Toxoplasma gondii acetylome showed that this PTM is abundant in this 
parasite; 411 lysine acetylation sites were detected across 274 tachyzoite proteins. The known acetylated marks on histones and $\alpha$-tubulin were detected and also a wide variety of acetylation sites in additional proteins. The majority of acetylation occurs on proteins involved in metabolism, translation, stress response, and chromatin biology [23]. An unpublished preliminary characterization performed by western blot indicates that acetylation is as widespread in $T$. cruzi as it is in T. gondii (unpublished results from our lab).

Finally we would like to mention bromodomains, the only known protein modules capable of recognizing acetyl lysine residues. This association regulates protein-protein interactions similar to how SH2 (Src homology 2) and PTB (phosphotyrosine-binding) modules bind to and regulate phosphotyrosine-containing proteins [66]. There are several coding sequences for bromodomain-containing factors in the Tritryps genomes [31]. Bromodomain factor 2 from T. cruzi (TcBDF2) has been characterized. It displays a nuclear localization in all life cycle stages and associates with acetylated histones $\mathrm{H} 2 \mathrm{~A}$ and $\mathrm{H} 4$ (having preference for $\mathrm{H} 4 \mathrm{~K} 10 \mathrm{ac}$, and probably $\mathrm{H} 4 \mathrm{~K} 14 \mathrm{ac}$ residues). TcBDF2 accumulation in epimastigotes after UV irradiation suggests a possible role for this protein in DNA repair [67]. Two more nonnuclear BDFs are currently under investigation. The presence of several putative bromodomain-containing proteins in Tritryps with different subcellular localizations supports the idea that these early-branched eukaryotes have established a network capable of recognizing lysine acetylation signals.

\section{Lysine Acetyltransferases}

Histone acetyltransferases (HATs) or lysine acetyltransferases (KATs) are evolutionarily conserved from yeast to humans although there is little similarity between the protein sequences of different members of this group. Many of them contain acetyl-CoA-binding domains as well as bromodomains and they form multisubunit complexes [68]. They are diverse in structure, substrate specificity, and function. Also, their classification is less clear in contrast to what happens with KDACs [65], which will be addressed latter.

KATs can be classified in two groups or general classes (A type and B type), depending on their intracellular localization and substrate specificity. A-type KATs are mainly nuclear and are involved in transcription-related acetylation as well as modification of core histones. These acetyltransferases can be further grouped into three main families (GNAT, p300/CBP, and MYST), based on sequence conservation of the HAT domain and biological functions. B-type KATs have a cytoplasmatic localization, where they acetylate free histone substrates and promote their nuclear localization. KAT families have high-sequence similarity within them but poor to no sequence similarity between any two given families [69].

The first KATs reported fifteen years ago were yeast Hat1 (histone acetyltransferase 1) and Gcn5 (general control nonrepressed 5), both members of the GNAT (Gcn5-related $\mathrm{N}$-acetyltransferases) family. These enzymes are important for telomeric silencing and transcriptional initiation, respectively [70]. The second family members, CBP (CREB binding protein) and p300, are part of the same protein complex. Also, p300 contains a bromodomain and acts on histone and nonhistone substrates. The MYST (MOZ, Ybf2/Sas3, Sas2, Tip60) family members function as catalytic subunits in Tip60 (60 kDa Tat-interactive protein), HBO1 (histone acetyltransferase binding to ORC 1), and $\mathrm{MOZ} / \mathrm{MOF}$ (monocytic leukemia zinc finger protein/male absent on first) complexes [65]. MYST acetyltransferases have been shown to participate in transcription, DNA replication, recombination and repair, cell cycle, and gluconeogenesis. They are generally part of multisubunit complexes in vivo and regulate themselves by autoacetylation [71].

Trypanosomatids encode few acetyltransferases, methyltransferases, and binding modules (e.g., bromodomains) compared to other eukaryotes. The Tritryps genomes contain six histone acetyltransferases [31]. KATs from trypanosomatids are very divergent when compared to those from mammals or yeast and do not contain any other recognizable binding domains. Four of them are related to the MYST family and two are of the elongator type, which belong to the GNAT family. When compared to other species, the Tritryps enzymes are only slightly conserved and not all of them have the exact same genes.

MYST family acetyltransferases are defined by a distinctly conserved domain which contains a C2HC $\left(\mathrm{CX}_{2} \mathrm{CX}_{12} \mathrm{HX}_{3-5} \mathrm{C}\right)$ zinc finger domain and an acetylCoA-binding site (motif $\mathrm{A}, \mathrm{Q} / \mathrm{RX}_{2} \mathrm{GXG} / \mathrm{A}$ ), homologous to that present in the GNAT family. Some members contain additional features such as chromodomains, PHD (Plant Homeo Domain) fingers and zinc fingers [71]. Trypanosomatids MYST acetyltransferases are quite divergent relative to members of this family in other organisms. Despite such divergence, there are common features present in Tritryps (Figure 1). For example, motif A (green boxes in Figure 1) and the critical residues for catalysis are conserved (asterisks in Figure 1). Also, a C2HCzinc-binding domain is present (grey box in Figure 1) and is thought to be involved in catalytic activity and/or substrate recognition [72]. But, there are some unique features among trypanosomatids KATs. For example, in KAT2 the motif A and the zinc-binding motifs are absent. HAT4 from T. cruzi and Leishmania have insertions of variable size and number in the acetyltransferase domain, and an important residue for catalysis is changed from $\mathrm{E}$ to $\mathrm{S}$ (asterisks in Figure 1). Also, there is a chromodomain present in HAT1 and 2 in all three Tritryps suggesting a link between acetylation and methylation in these protozoan parasites.

In Table 2 we summarize the KATs present in trypanosomatids and their known functions, which are detailed next. Trypanosoma brucei contains three KATs that belong to the MYST family. Siegel et al. demonstrated that TbKAT3 is a nuclear protein that is responsible for acetylation of $\mathrm{H} 4 \mathrm{~K} 4$, a modification considered more structural than regulatory. Also, they observed that treatment with cycloheximide, an inhibitor of protein synthesis, leads to a complete loss of unmodified H4 K4 sites. These facts suggest that newly synthesized histone $\mathrm{H} 4$ with unmodified $\mathrm{K} 4$ is 


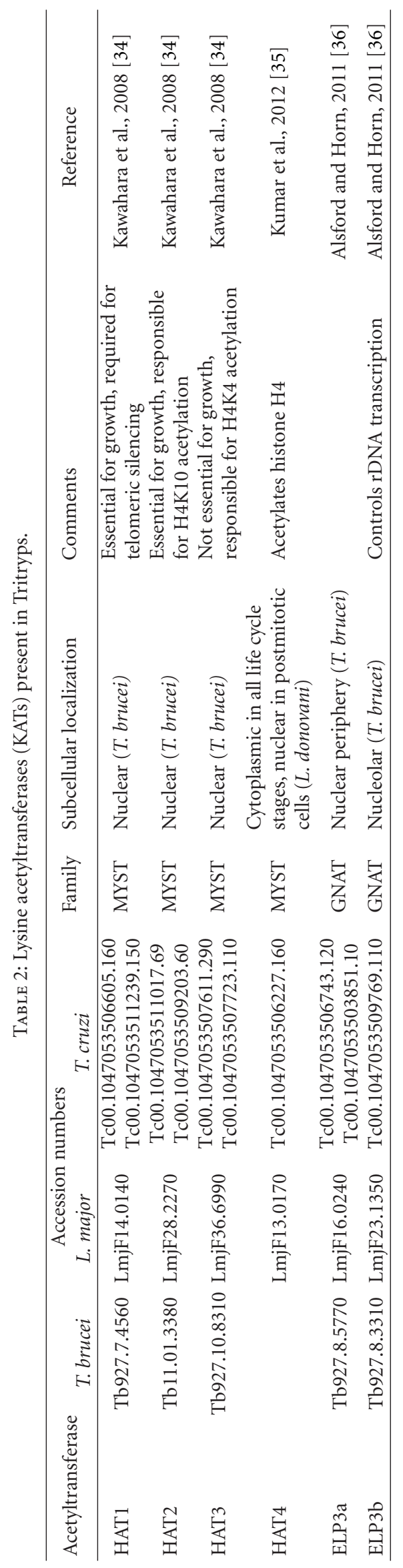



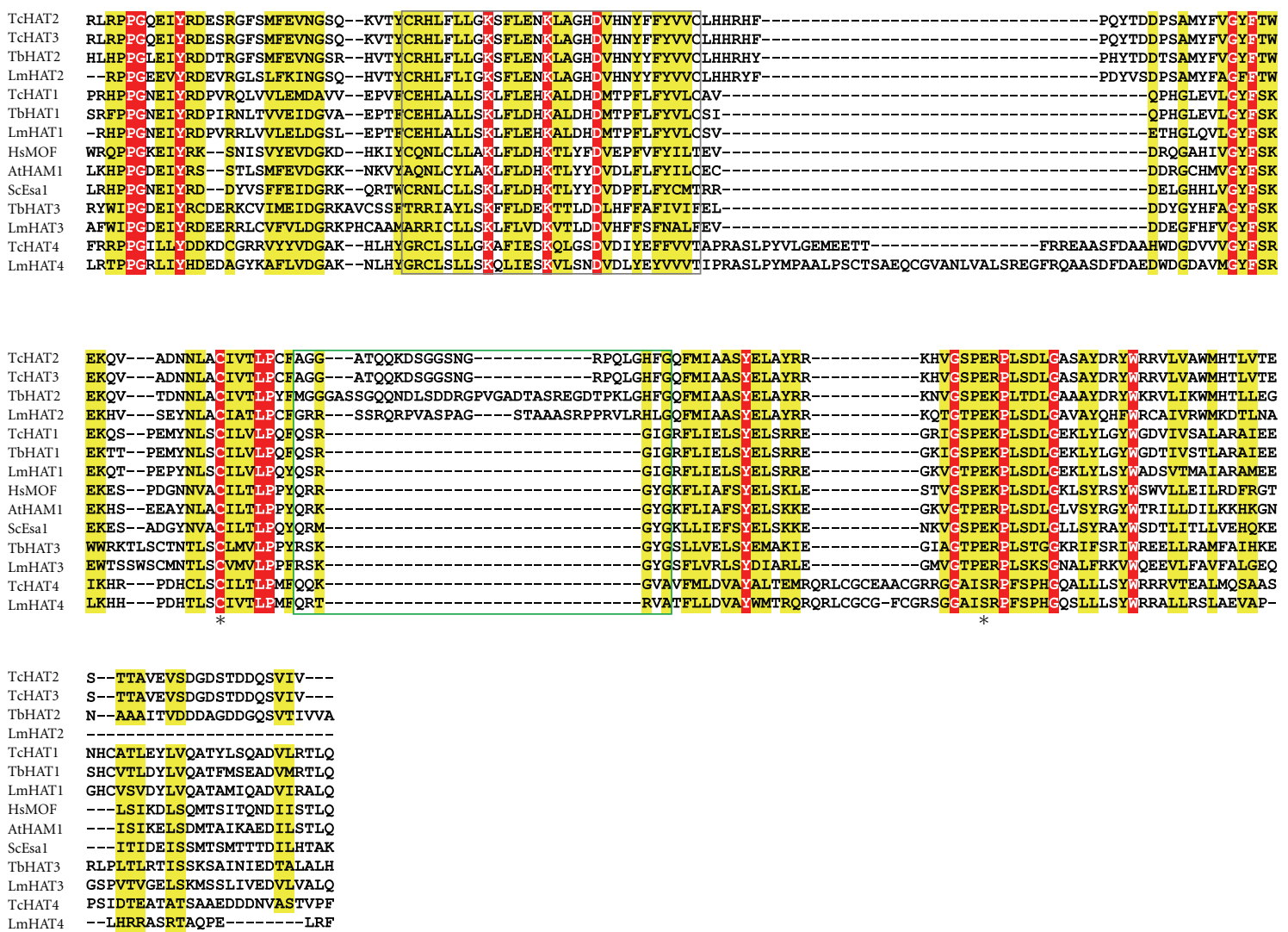

FIGURE 1: MYST family histone acetyltransferase orthologues in trypanosomatids. Multiple sequence alignments of the core acetyltransferase domains from Tritryps and other organisms. Sequences were aligned with ClustalX2 followed by manual adjustment and illustrated with ESPript 2.2. Identical residues are white on red background, and residues shared by some organisms are black on yellow background. Asterisks indicate residues that were predicted to form part of the catalytic pocket. The gray box marks the C2HC zinc-binding domain. Motif A that binds acetyl Co-A is indicated with a green box. Tb, Trypanosoma brucei; Tc, Trypanosoma cruzi; Lm, Leishmania mayor; At, Arabidopsis thaliana; Hs, human; Sc, Saccharomyces cerevisiae; AcuC, acetoin utilization.

rapidly imported to the nucleus where it suffers a possibly irreversible acetylation. The authors also suggest that $T$. brucei may not deacetylate histones recently synthesized in the cytoplasm as other eukaryotes do and that elevated levels of H4 K4 acetylation might serve to keep chromatin in an open conformation [73]. Another report from the same year demonstrated that TbKAT3 is dispensable, while TbKAT1 and 2 are required for parasite growth [34]. KAT4 coding sequence is not present in the T. brucei genome, but it is present in T. cruzi and Leishmania [31]. All three $T b K A T s$ are present in the nucleus of bloodstream and insectstage cells and mRNA abundance experiments revealed no difference in their expression levels. TbKAT1 knock-down cells have telomeric silencing specifically compromised, and this enzyme might be required for DNA replication. TbKAT2 displays in vitro acetyltransferase activity towards H4 K10. Apparently, trypanosomes use specific MYST acetyltransferases to acetylate $\mathrm{H} 4 \mathrm{~K} 4$ and $\mathrm{H} 4 \mathrm{~K} 10$, which further supports the idea of a nonredundant histone code present in these parasites [34]. Leishmania donovani KAT4 was recently characterized. This protein has a cytoplasmatic localization throughout the parasite life cycle and a nuclear localization in postmitotic cells. LdKAT4 acetylates histone
$\mathrm{H} 4$ at $\mathrm{K} 4$ residues almost exclusively and $\mathrm{K} 2$ in a minor proportion. These results suggest that $\mathrm{H} 4$ modification might happen in the cytoplasm prior to histone transport to the nucleus or soon after mitosis inside the nuclear compartment [35]. All this evidence clearly illustrates that KATs seem to be equivalent among the three genera but play completely different roles in each organism. Moreover, these differences may reflect that they are involved in distinct cellular processes. The most obvious example of this is the fact that the nuclear TbKAT3 is playing the same role of the cytoplasmatic LdKAT4, they both acetylate $\mathrm{H} 4$ at K4. If this is true, the mechanism by which newly synthesized histone $\mathrm{H} 4$ is acetylated differs between these species not only due to the participation of different enzymes but due to the fact that it occurs in different cellular compartments.

The GNAT family has sequence homology limited to 4 motifs (A-D) of 15-33 amino acids each. The central core domain is structurally conserved in different organisms and interacts with coenzyme A (CoA) through motif A which plays an important role in catalysis [74]. The members of this family present in trypanosomatids are two Elp3 (Elongator protein 3) orthologues which contain a GNAT-related acetyltransferase domain. In mammals, the elongator complex 
has six subunit (Elp1-6), and it was first identified due to its association with RNAP II and its role in transcriptional elongation. One of the subunits of the elongator complex, Elp3, has a characteristic GNAT acetyltransferase domain is capable of acetylating both histones and other nonhistone substrates and is conserved from archaea to humans. In fact, the Elongator complex is mainly present in the cytoplasm where it is involved in stress signaling, exocytosis, tRNA modification, and $\alpha$-tubulin acetylation (reviewed in [75]). Trypanosomatids Elp3a and Elp3b sequences have an ironsulfur cluster (radical S-adenosylmethionine or SAM, grey boxes on Figure 2) and the motif A (green boxes in Figure 2). Despite the divergence with orthologues from other organisms the amino acids required for substrate binding are conserved (asterisks and arrowheads in Figure 2). It is worth noting that only Tritryps have two Elp3 orthologues, another unique feature that may reflect distinct roles for these organisms enzymes.

A recent report by Alsford and Horn describes that the Elongator proteins (Elp3) present in T. brucei display acetyltransferase activity. TbElp3a localizes at the nuclear periphery, while $T b E l p 3 b$ is concentrated in the inner part of the nucleus in both bloodstream and insect-stage cells. Using null mutants these authors showed that $T b E l p 3 b$ negatively controls transcription elongation and rDNA transcription [36]. These results indicate that all KATs from T. brucei are exclusively nuclear and mainly related to transcription or other nuclear processes, suggesting that at least one not yet described enzyme should be responsible for the acetylation of cytoplasmic proteins as occurs in other organisms. Preliminary results from our lab using GFP fusions in T. cruzi suggest that Elp3a and Elp3b are not nuclear proteins.

Finally, we would like to mention another acetyltransferase reported in 2010, named MEC-17. This KAT is a member of the GNAT family and behaves as a K40specific acetyltransferase for $\alpha$-tubulin in Tetrahymena and Caenorhabditis elegans. This enzyme contributes to touch sensation and probably acetylates other nontubulin substrates in both organisms. MEC-17 homologues are present in most eukaryotes with the exception of fungi and plants [76]. In trypanosomatids there are several hypothetical proteins similar to MEC-17, none of which have been characterized yet.

\section{Lysine Deacetylases}

Mammalian and yeast KDACs can be divided into two families: classical or zinc dependent and $\mathrm{NAD}^{+}$-dependent. Another classification divides all KDACs into four classes according to their phylogenetic conservation. Classes I, II, and IV belong to the classical family, while class III contains $\mathrm{NAD}^{+}$-dependent deacetylases, also termed sirtuins (SIRTs) because of their homology with yeast SIR2 proteins (silent information regulator 2). Class I and II deacetylases are represented by Saccharomyces cerevisiae Rpd3-like (reduced potassium dependency 3 ) and Hda1-like (histone deacetylase 1) proteins, respectively. The members of these classes have overlapping but distinguishable roles. In humans, class I is represented by HDAC1, 2, 3, and 8; class II is represented by HDAC4, 5, 6, 7, 9, and 10 and Class IV is represented by HDAC11. Class III (or Sirtuins) has seven members (SIRT17) [77], which are present from bacteria to humans. There are nuclear and cytoplasmic KDACs and only sirtuins are present in the mitochondria [78]. For example, mammalian HDAC6 is a tubulin deacetylase that regulates cell motility [79].

In Table 3 we summarize the KDACs present in trypanosomatids and their known functions. Again, the number of enzymes is small in comparison to mammals. The number of KDACs is reduced to only four enzymes and three SIR2-related proteins (SIRT2 RP). Tritryps class I KDACs have $350-500$ amino acid residues $(\sim 50 \mathrm{kDa})$, a deacetylase domain and a small C-terminal region that can suffer several PTMs. Figures 3 and 4 show sequence alignments of the class I and II deacetylase domain in different organisms. Classspecific motifs are indicated with black boxes and residues important for catalysis and binding zinc-cofactor are marked with asterisks and triangles, respectively. Apparently, all class I and II KDACs have insertions of variable length in different positions.

In T. brucei DAC1 and 2, both members of class I KDACs, are orthologues of Rpd3 from S. cerevisiae. DAC2 has a highly acidic C-terminal domain that might be involved in proteinprotein interaction or cellular localization. This is an unusual protein because it lacks several pocket residues, which are predicted to be required for deacetylase activity (asterisks in Figure 3). Ingram and Horn, through disruption of DAC genes in bloodstream T. brucei cells, determined that DAC1 is essential, while DAC2 is dispensable. Class II KDACs (DAC3 and DAC4) are larger than their class I counterparts, with an Hda1-catalitic domain at the $\mathrm{C}$-terminus and other domains and regulatory sites at the N-terminus. In T. brucei DAC3 and DAC4 are ScHdal orthologues, and only the former is essential. TbDAC4 mutants display a delay in the G2/M phase of the cell cycle. Based on these features the authors propose that TbDAC1 and TbDAC3 might be potential chemotherapeutic targets [46]. Wang et al. went further with the characterization of these deacetylases and reported that DAC1 and DAC3 are nuclear proteins that display deacetylase activity and are essential for growth in the bloodstream forms of T. brucei. TbDAC1 antagonizes telomeric silencing and TbDAC3 is required for variant surface glycoprotein expression sites (VSG ESs) silencing in bloodstream and insect stage cells [47]. The same report states that DAC2 and 4 are predominantly cytoplasmatic. Detailed information about localization and function of DACs from Leishmania and T. cruzi is still lacking. DAC2 seems to be absent in Leishmania; however, its function might be substituted by DAC1. The fact that TbDAC2 is not essential could support this hypothesis. There are two sequences available for DAC2 and DAC3 that correspond to alleles from Esmeraldo and non-Esmeraldo strains in T. cruzi.

Class III KDACs or sirtuins (SIRTs) are $\mathrm{NAD}^{+}$-dependent deacetylases that have diverse subcellular localization. They are involved in transcriptional silencing, DNA repair, cell cycle progression, chromosome segregation, and life span [80]. Members of the SIRT family have a 250 amino acid core domain, which exhibits a $25-60 \%$ sequence identity in different organisms. SIRTs remove acetyl groups 


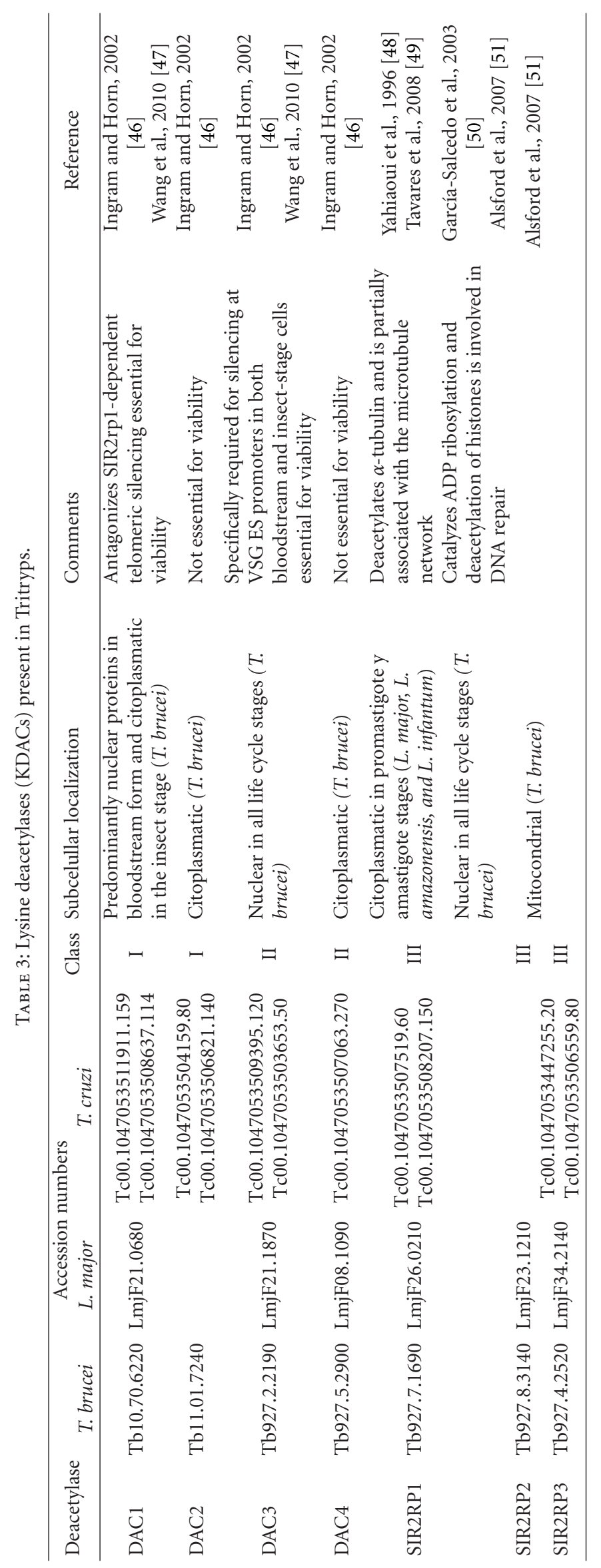



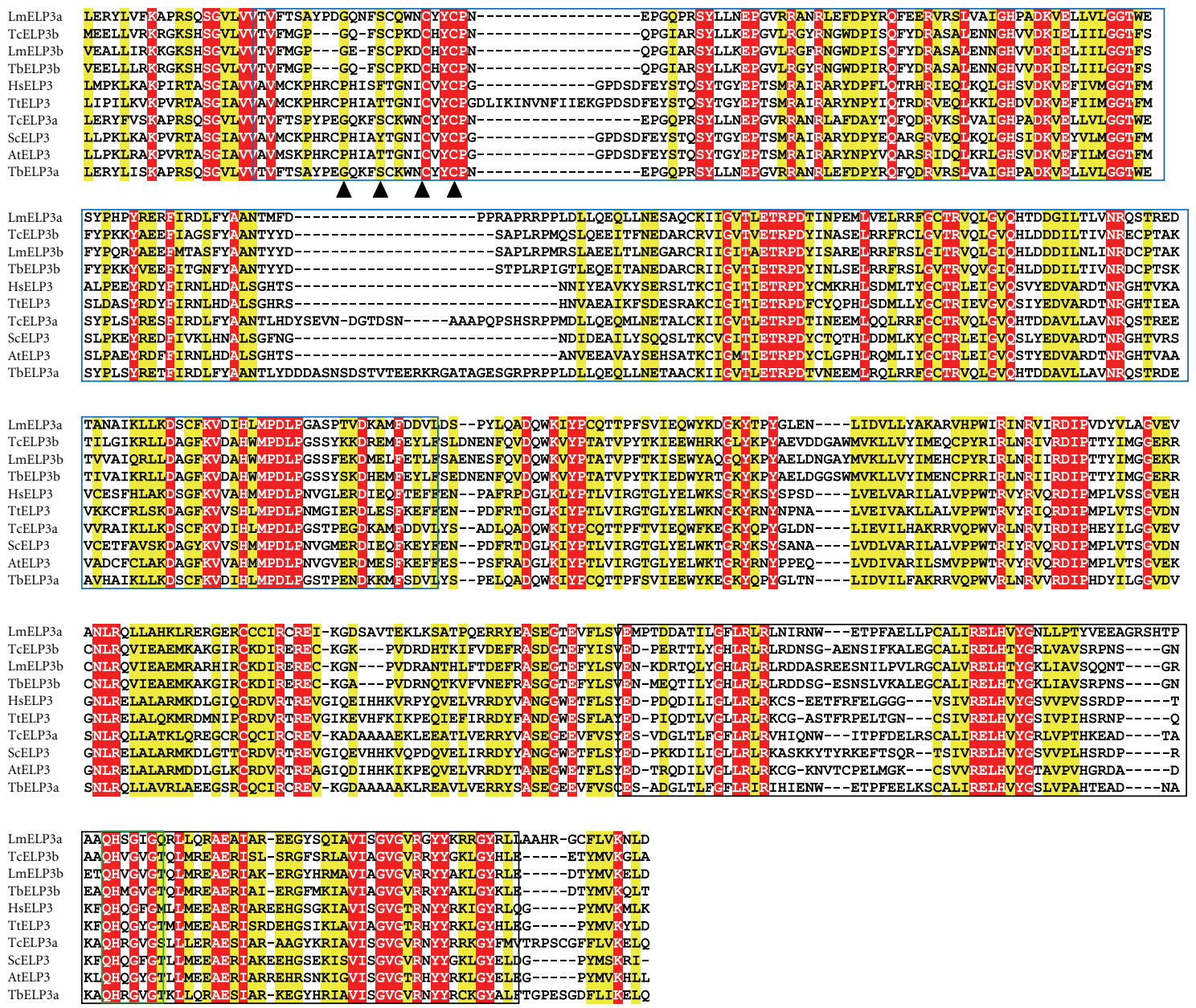

FIgURE 2: GNAT family histone acetyltransferases orthologues in trypanosomatids. Multiple sequence alignments of the core acetyltransferase domains from Tritryps and other organisms. Sequences were aligned with ClustalX2 followed by manual adjustment and illustrated with ESPript 2.2. Identical residues are white on red background, and residues shared by some organisms are black on yellow background. Radical SAM domain (blue box) and GNAT-type acetyltransferase domain (black box) are indicated. Motif A is marked with a green box. Arrowheads indicate the Cys residues that are part of the Fe-S cluster in the SAM domain. Tb, Trypanosoma brucei; Tc, Trypanosoma cruzi; Lm, Leishmania mayor; At, Arabidopsis thaliana; Hs, human; Sc, Saccharomyces scerevisiae: Tt, Tetraymena thermophila.

in nuclear, cytoplasmatic, and mitochondrial substrates. During the deacetylation reaction, acetyl-lysine and $\mathrm{NAD}^{+}$ are converted into lysine, nicotinamide and O-acetyl-ADPribose [81]. Michishita et al. characterized seven human proteins homologous to Sir2 and concluded that (1) three nuclear SIRT proteins (SIRT1, SIRT6, and SIRT7) show different subnuclear localizations, being SIRT6 and SIRT7 associated with heterochromatic regions and nucleoli; (2) SIRT3, SIRT4, and SIRT5 are localized in mitochondria, an organelle linked to aging and energy metabolism; (3) cellular p53 is a major in vivo substrate of SIRT1 deacetylase, but not of the other six SIRT proteins; (4) SIRT1, but not the other two nuclear SIRT proteins, shows an in vitro deacetylase activity on histone $\mathrm{H} 4$ and p53 peptides; (5) overexpression of the seven SIRT proteins does not extend cellular replicative lifespan in normal human fibroblasts or prostate epithelial cells [82]. Additionally, hSIRT2 deacetylates $\alpha$-tubulin [83] and shuttles to the nucleus during G1/M phase transition regulating microtubule dynamic and cell cycle progression
[84]. Sirtuins from T. brucei, cruzi, and Leishmania are listed in Table 3 along with their known functions.

There are three SIRP2RPs in trypanosomatids, one on them (SIR2 RP2) is not present in T. cruzi. All trypanosomatid SIR2RPs lack the N-terminal portion of ScSir2, which is required for nucleolar localization, but all contain a complete catalytic domain (Figure 5). RP1s contain serinerich motifs towards the C-terminus and RP2s have two insertions in Tritryps. All trypanosomatid proteins have a zinc-binding motif $\left(\mathrm{CX}_{2} \mathrm{CX}_{20} \mathrm{CX}_{2} \mathrm{X}\right.$ type), but one of the Cys residues is absent in the three SIR2 RP3 (grey box in Figure 5). Apparently all SIR2 RP2 from Tritryps are phylogenetically closer to bacterial SIRTs, while RP1 and RP3 are more related to yeast and human SIRTs, respectively [51]. In all trypanosomatid sirtuins two motifs (GAG and NID) and two specific residues (HG) essential for enzymatic activity are conserved (underlined in Figure 5).

L. mayor SIR2 RP1 was the first sirtuin homologue described in a kinetoplastid protozoa [48] and is localized 


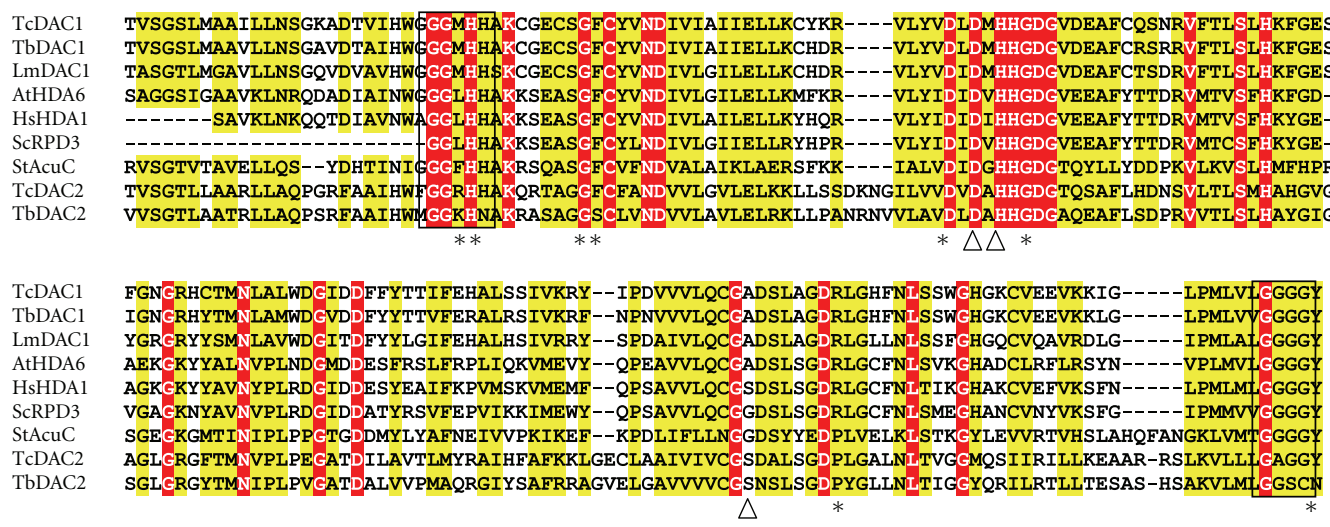

Figure 3: Class I deacetylases orthologues in Trypanosomatids. Alignment of core deacetylase domain from different organisms. Sequences were aligned using ClustalX and were adjusted manually and illustrated with ESPript 2.2. The black boxes indicate the class specific motifs. Identical residues are white on red background and residues shared by some organisms are black on yellow background. Asterisks indicate residues predicted to form part of the catalytic pocket whereas triangles indicate zinc cofactor ligands. Tb, Trypanosoma brucei; Tc, Trypanosoma cruzi; Lm, Leishmania mayor; At, Arabidopsis thaliana; Hs, human; Sc, Saccharomyces cerevisiae; St, Sulfolobus tokodaii.

in cytoplasmatic granules. Leishmania infantum SIR2 RP1 is expressed in each developmental stage, it contains the conserved core domain and is a $\mathrm{NAD}^{+}$-dependent deacetylase and ADP-ribosyltransferase. Also, LiSIR2 RP1 is cytoplasmatic, similar to human SIRT2 [83] and HDAC6 [79]. It associates with the cytoskeleton network and deacetylates $\alpha$-tubulin [49]. Leishmania amazonensis Sir2 RP1 is expressed in cytoplasmatic vesicles of both promastigote and amastigote developmental forms. Its secretion/excretion was correlated with the glycosylation state of the protein [85]. TbSIR2 RP1 is a nuclear and chromosome-associated protein. It is expressed throughout T. brucei life cycle and catalyses $\mathrm{NAD}^{+}$-dependent $\mathrm{ADP}$ ribosylation and deacetylation of histones as some human SIRTs do $[50,86]$. Overexpression of TbSIR2RP1 is toxic to the T. brucei bloodstream form but not to the insect stage parasite. Moreover, it controls DNA repair and repression of RNA Pol I-mediated expression immediately adjacent to telomeres, but it is not required for antigenic variation [51]. TbSIR2 RP2 and 3 were identified in the single mitochondrion of the parasite. These genes were disrupted, but the mutant strains did not show growth or differentiation defects [51]. The fact that T. brucei and Leishmania have mitochondrial deacetylases makes sense considering that acetylation is very important for the regulation of the energetic metabolism, as demonstrated in other organisms. It also highlights the need of an acetyltransferase activity out of the nucleus in T. brucei, suggesting that at least one of the nuclear KATs mentioned above might have a dual nuclear-cytoplasmatic localization as some mammalian enzymes have.

\section{KDACs and KATs Inhibitors as Chemotherapeutic Agents}

In the last few years, a number of KDAC and KAT inhibitors (iKADCs and iKATs) were developed and assayed against several types of cancer where these enzymes are deregulated and generally overexpressed [87]. KATs and KDACs inhibitors are now being investigated to target a range of parasitic diseases with the advantage that some of these compounds have already been validated for other human diseases and the chemical starting points are already available. Also, these compounds are very useful tools to study the catalytic features of acetyltransferases and deacetylases, as well as their roles in different cellular pathways. KDAC inhibitors were known before the discovery of their target enzymes. For example, sodium butyrate, trichostatin A, and valproic acid were described to induce mammalian histone acetylation many years ago [88-90]. These compounds have diverse structures and mechanisms of action for inhibiting their target proteins. Inhibition of KDACs has multiple effects on the cell, such as cell cycle arrest, senescence, apoptosis, ROS production, and mitotic cell death. iKDACs are capable of reducing tumor invasiveness, angiogenesis, and metastasis in vivo (reviewed in [91]). Sirtuins inhibitors were also described many years ago, for example, sirtinol [92-95] and nicotinamide, which is a product of the Sir2-catalyzed reaction [96]. iKATs are less studied that iKDACs and only a small number of them are known, such as curcumin, anacardic acid, garcinol, and isothiazolones. iKATs have different target proteins and cell permeability. Recently, there have been advances in the development of cell-permeable small molecules that behave as specific modulators of HATs (reviewed in [97]).

As we stated before, current knowledge leads to think that KATs and KDACs are crucial in the life cycle of trypanosomatids, thus these enzymes could be interesting chemotherapeutic targets for treating parasitic diseases. Several inhibitors have been tested against protozoan parasites in vitro and in vivo, but there are still many critical factors that need to be considered before using these compounds clinically. Next, we describe the iKATs and iKDACs that have been tested against protozoa, focusing on trypanosomatids (Table 4). 

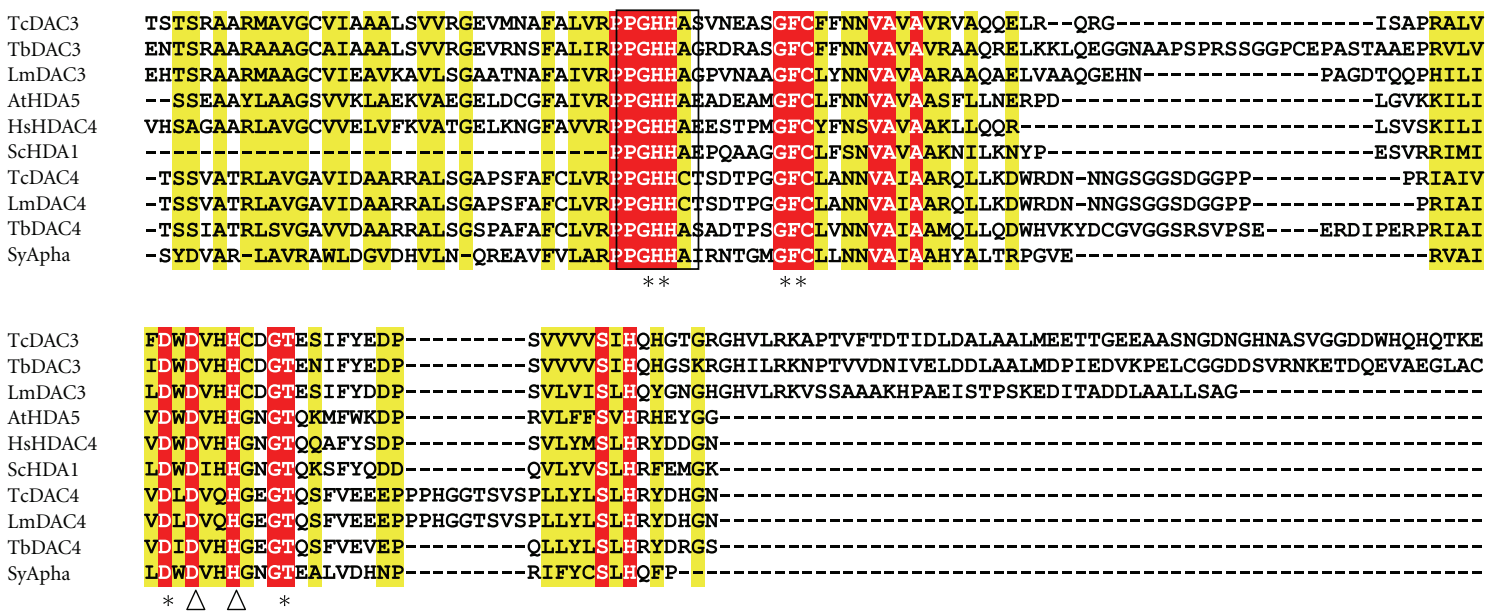

DEETEEEEEAENAEKVQRQNPQISPEEEGDGRKRARR- KPVDYVKLAADIT TCDAC3 3 EEEEEEEEAENAEKVQRQNPQLSPEEEGDGRKRAARR--KPVDYVKLAADLE-EDDEAIARMFGISPEDLKKSSSSFSMSKSSDGGGGGSSGSRSS
TbDAC3 GTVPNERSPVKEEESTGGECKHESPQDDEEKKESSCRRQRKPVDYNKLAEEMA-NODDELARIFGVNAEELAR----FPES--------GSSGSESD LmDAC3 --TIEPPPVSA PEASRPEAASDPPQDSTEG-----RRVRAAVDYNSLAAQIEKEDDAEIAALFGVDLNTASSSSSNSSSAS-------NSSSVSAD

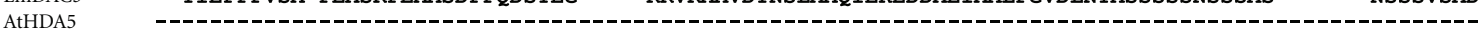
AtHDA5 1 HsHDAC4 ScHDAl TcDAC4 LmDAC4 TbDAC4 SyApha

TcDAC3 TbDAC3 LmDAC3 AtHDA5 HsHDAC4 ScHDAl TcDAC4 LmDAC4 TbDAC4 SyApha

TcDAC3 TbDAC3 LmDAC3 AtHDA5 HsHDAC4 ScHDAl TCDAC4 LmDAC4 TbDAC4 SyApha

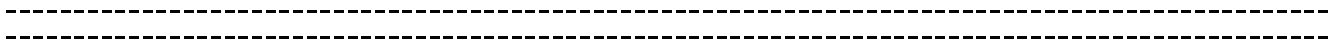

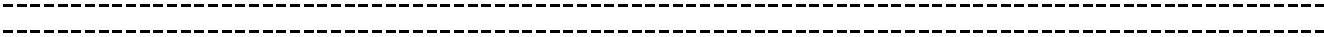
-

SSHFYSAHLVQKNLPGDTEGLSLDED-----IDNDKDGKFYPG--TGHVERVGGDTRAEARGKNINI PWPTLG---MGDLEYLQVFLDIVAPVVREY SSTSSESOERAGKLSGDSEGYSYSDE-----DSSNEPRPFYPS--TEHMDRVGGDANPEAKGKNINIPWPTHN---MGDLEYLOVVLDVVLPVMREF STSVARNDTRPVHYAGDTVGLSFDETPLQRATGTEEEELFYPG--TGHLNRVGGDATPAAQGRNINI PWPTHG---MGDLEYLQLLHEVVLPAAREF -

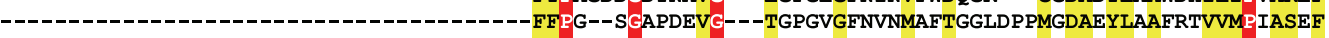
作 作

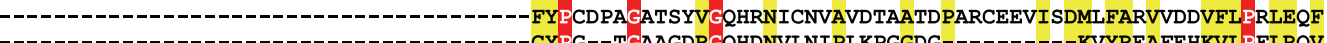

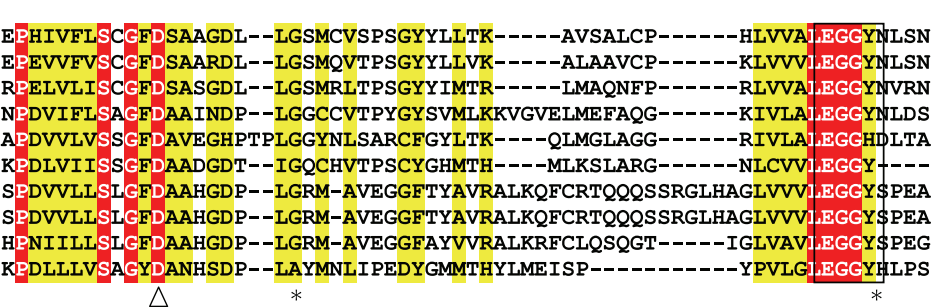

FIgURE 4: Class II deacetylases orthologues in trypanosomatids. Alignment of the core deacetylase domain from different organisms. Sequences were aligned using ClustalX and were adjusted manually and illustrated with ESPript 2.2. The black boxes indicate the classspecific motif. Identical residues are white on red background, and residues shared by some organisms are black on yellow background. Asterisks indicate residues that were predicted to form part of the catalytic pocket, whereas triangles indicate zinc cofactor ligands. Tb, Trypanosoma brucei; Tc, Trypanosoma cruzi; Lm, Leishmania mayor; At, Arabidopsis thaliana; Hs, human; Sc, Saccharomyces cerevisiae; Sy, Synechocystis spp.; AphaA, acetylpolyamine aminohydrolase.

Apicidin (iKDAC) displays antiparasitic activity against apicomplexan parasites such as Plasmodium falciparum and Toxoplasma gondii, among others [98]. An apicidin analogue has been reported as having a potent and selective activity against T. brucei but is toxic to L. donovani and T. cruzi. This species-specific effect observed for Trypanosoma species was also seen for three other synthetic analogues [99]. Valproic acid, sodium butyrate and its derivatives all have quite poor in vitro activity against $P$. falciparum and $T$. gondii but are useful for studying the acetylation-dependant cellular pathways [100, 101]. Nicotinamide (nam) has antiparasitic activity against several Leishmania species [102]. Moreover, nam and trichostatin A (TSA) cause growth arrest in T. cruzi epimastigotes at micromolar concentrations (unpublished results from our lab). TSA and SAHA (suberoylanilide hydroxamic acid) are both hydroxamate-based compounds that inhibit T. gondii and P. falciparum growth in vitro, but they kill mammalian cells at similar concentrations $[100,103]$. Very little information is available on the activity of hydroxamate or other HDAC class I or II inhibitors against Tritryps. However, as stated for other compounds, TSA is a very useful tool that helps understand how KDAC inhibition affects the parasite growth, development, and transcriptional control. Sirtinol, a Sir2 inhibitor, which is 
TABLE 4: KDACs and KATs inhibitors tested in protozoan parasites.

\begin{tabular}{|c|c|c|c|}
\hline & Structure & Target & Antiparasitic activity \\
\hline \multicolumn{4}{|l|}{ KDAC inhibitors } \\
\hline Apicidin & Cyclic tetrapeptide & All KDAC & Apicomplexa T. gondii, P. falciparum \\
\hline Apicidin analogues & $\begin{array}{l}\text { Cyclic tetrapeptide with indole } \\
\text { modifications }\end{array}$ & All KDAC & T. gondii, P. falciparum, T. brucei \\
\hline Valproic acid & Short-chain fatty acid & Class I KDAC & P. falciparum, T. gondii \\
\hline Sodium butyrate & Short-chain fatty acid & All KDAC & P. falciparum, T. gondii \\
\hline Sirtinol & $\begin{array}{l}\text { 2-hydroxy-1-napthaldehyde } \\
\text { derivative }\end{array}$ & SIR2 & $\begin{array}{l}\text { L. infantum amastigotes, } P \text {. falciparum, } \\
\text { T. gondii }\end{array}$ \\
\hline Nicotinamide & Noncompetitive inhibitor & SIR2 & Leishmania spp., T. cruzi epimastigotes \\
\hline Tricostain A (TSA) & Natural hydroxamate based & Class I and II KDAC & T. cruzi, P. falciparum \\
\hline $\begin{array}{l}\text { Suberoylanilide } \\
\text { hydroxamic acid } \\
\text { (SAHA) }\end{array}$ & Synthetic hydroxamate based & All KDAC & P. falciparum \\
\hline $\begin{array}{l}\text { Hydroxamic acid } \\
\text { derivatives }\end{array}$ & & All KDAC & T. brucei \\
\hline \multicolumn{4}{|l|}{ KAT inhibitors } \\
\hline Curcumin & Natural phenol & MYST family p300/CBP & T. brucei, L. mexicana \\
\hline Garcinol & Polyisoprenylated benzophenone & $\begin{array}{l}\text { MYST family p300 and } \\
\text { PCAF }\end{array}$ & Leishmania spp. \\
\hline Anarcadic acid & $\begin{array}{l}\text { Salicylic acid substituted with an } \\
\text { alkyl chain }\end{array}$ & $\begin{array}{l}\text { MYST family p300 and } \\
\text { PCAF, Tip60 }\end{array}$ & P. falciparum \\
\hline
\end{tabular}

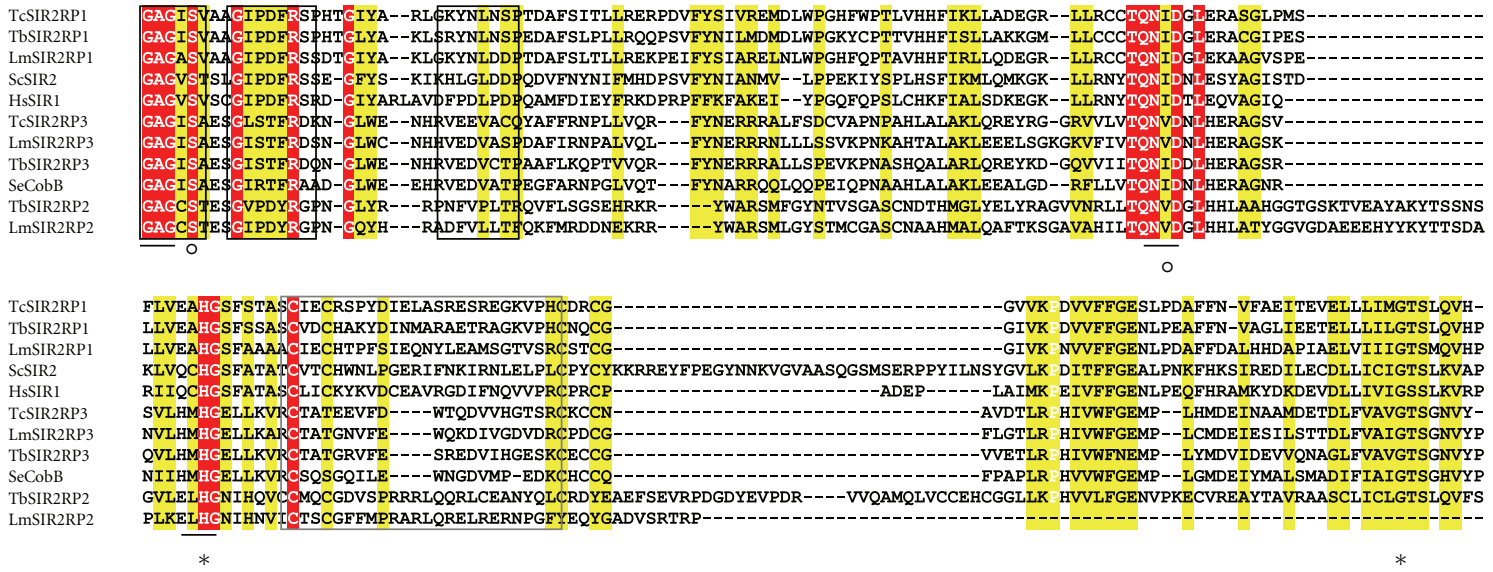

FIGURE 5: Class III deacetylases orthologues in trypanosomatids. Multiple sequence alignments of the core deacetylase domains from Tritryps and other organisms. Sequences were aligned with ClustalX2 followed by manual adjustment and illustrated with ESPript 2.2. Identical residues are white on red background and residues shared by some organisms are black on yellow background. Asterisks indicate residues that were predicted to form part of the catalytic pocket. The grey box marks the $\mathrm{C} 2 \mathrm{HC}$ zinc-binding domain. Circles indicate $\mathrm{NAD}^{+}$-binding residues and asterisks indicate catalytic residues. The GAD and NID motifs as well as the conserved HG residues important for catalysis are underlined. Tb, Trypanosoma brucei; Tc, Trypanosoma cruzi; Lm, Leishmania mayor; At, Arabidopsis thaliana; Hs, human; Sc, Saccharomyces cerevisiae; Sy, Synechocystis spp.

commercially available, is capable of inducing death in $L$. infantum amastigotes [104]. Also, it was found to inhibit growth in P. falciparum [105]. A number of hydroxamic acid derivatives, which inhibit human deacetylases, were recently tested against T. brucei bloodstream form. Most of the derivatives assayed, identified by screening compound libraries, were able to block parasite growth in the submicromolar range. The most effective compound was a member of the sulphonepiperazine series [106].

As we mentioned before, only a small number of iKATs have been reported, and even fewer have been tested against protozoan parasites. For example, curcumin has trypanosidal 
activity in vitro $[107,108]$ and curcumin analogues have a potent activity against T. brucei bloodstream forms and L. mexicana amastigotes and promastigotes in vitro [109]. Anacardic acid blocked growth of $P$. falciparum in vitro by reversibly and noncompetitively inhibiting the KAT activity of recombinant $P f \mathrm{GCN} 5$ [110]. Other natural acetyltransferase inhibitors such as, garcinol and xhantanes were tested in vitro in Leishmania, the results were promising, but the cytotoxicity profile of these compounds needs to be assessed before evaluating them in vivo [111].

All these evidence supports the idea that KDACs and KATs are promising drug targets against protozoan parasites and it would be extremely valuable to fully characterize these enzymes and discover their mechanisms of action in order to asses future inhibitors. As we mentioned before, these compounds are also useful to study localization, stability and interactions of deacetylases and acetyltransferases in trypanosomatids.

\section{Concluding Remarks}

The flagellated protozoa T. brucei, T. cruzi, and Leishmania spp. represent important human and animal pathogens and are emerging as model organisms for the study of epigenetic regulation [112]. Several lines of evidence described here indicate that lysine deacetylases and acetyltransferases constitute key enzymes in trypanosomatids and some of them are essential for their viability. Even though the enzymes that exist in the three genera are considered orthologues, in some cases they do not have the same function and/or the same cellular localization. We observed that there is a tendency to extrapolate results from one species to other. In particular, from $T$. brucei, a parasite with many genetic tools including a very efficient iRNA system, to T. cruzi, which have limited genetic tools available. In the case of these enzymes, we highlight the need to study each organism separately to discover their mechanisms of action. Even though in many cases these extrapolations are correct, in the case of these enzymes we highlight the need to study each organism separately to discover their mechanisms of action. The search for KAT and KDAC inhibitors is a very active research field. There are several inhibitors described in the literature, which are very useful tools for unraveling the mechanisms of action and targets of these enzymes. Many of these compounds were assayed against parasites. The results presented here illustrate the fact that acetyltransferase and deacetylase inhibitors are species-specific in Tritryps and that most of these compounds inhibit mammalian enzymes as well. These results further support the need to study and fully characterize each trypanosomatid KAT and KDAC in order to rationally design new drugs to battle these pathogens.

\section{Acknowledgments}

The authors would like to thank Dr. Pamela Cribb and Carla Ritagliati for their valuable inputs and corrections.

\section{References}

[1] T. Kouzarides, "Acetylation: a regulatory modification to rival phosphorylation," EMBO Journal, vol. 19, no. 6, pp. 11761179, 2000.

[2] A. Norvell and S. B. McMahon, "Rise of the rival," Science, vol. 327, no. 5968, pp. 964-965, 2010.

[3] C. D. Allis, S. L. Berger, J. Cote et al., "New nomenclature for chromatin-modifying enzymes," Cell, vol. 131, no. 4, pp. 633-636, 2007.

[4] World Health Organization, http://www.who.int/en/ .

[5] S. Martínez-Calvillo, J. C. Vizuet-de-Rueda, L. E. FlorencioMartínez, R. G. Manning-Cela, and E. E. Figueroa-Angulo, "Gene expression in trypanosomatid parasites," Journal of Biomedicine \& Biotechnology, vol. 2010, Article ID 525241, 15 pages, 2010.

[6] W. De Souza, "Basic cell biology of Trypanosoma cruzi," Current Pharmaceutical Design, vol. 8, no. 4, pp. 269-285, 2002.

[7] K. R. Matthews, "The developmental cell biology of Trypanosoma brucei," Journal of Cell Science, vol. 118, no. 2, pp. 283-290, 2005.

[8] R. J. Wheeler, E. Gluenz, and K. Gull, "The cell cycle of Leishmania: morphogenetic events and their implications for parasite biology," Molecular Microbiology, vol. 79, no. 3, pp. 647-662, 2011.

[9] A. H. Kollien and G. A. Schaub, "The development of Trypanosoma cruzi in triatominae," Parasitology Today, vol. 16, no. 9, pp. 381-387, 2000.

[10] V. G. Allfrey and A. E. Mirsky, "Structural modifications of histones and their possible role in the regulation of RNA synthesis," Science, vol. 144, no. 3618, p. 559, 1964.

[11] D. M. Phillips, "The presence of acetyl groups of histones," The Biochemical Journal, vol. 87, pp. 258-263, 1963.

[12] B. D. Strahl and C. D. Allis, "The language of covalent histone modifications," Nature, vol. 403, no. 6765, pp. 41-45, 2000.

[13] T. Jenuwein and C. D. Allis, "Translating the histone code," Science, vol. 293, no. 5532, pp. 1074-1080, 2001.

[14] S. D. Taverna, B. M. Ueberheide, Y. Liu et al., "Longdistance combinatorial linkage between methylation and acetylation on histone $\mathrm{H} 3 \mathrm{~N}$ termini," Proceedings of the National Academy of Sciences of the United States of America, vol. 104, no. 7, pp. 2086-2091, 2007.

[15] S. C. Kim, R. Sprung, Y. Chen et al., "Substrate and functional diversity of lysine acetylation revealed by a proteomics survey," Molecular Cell, vol. 23, no. 4, pp. 607-618, 2006.

[16] B. J. Yu, J. A. Kim, J. H. Moon, S. E. Ryu, and J. G. Pan, "The diversity of lysine-acetylated proteins in Escherichia coli," Journal of Microbiology and Biotechnology, vol. 18, no. 9, pp. 1529-1536, 2008.

[17] J. Zhang, R. Sprung, J. Pei et al., "Lysine acetylation is a highly abundant and evolutionarily conserved modification in Escherichia coli," Molecular and Cellular Proteomics, vol. 8, no. 2, pp. 215-225, 2009.

[18] C. Choudhary, C. Kumar, F. Gnad et al., "Lysine acetylation targets protein complexes and co-regulates major cellular functions," Science, vol. 325, no. 5942, pp. 834-840, 2009.

[19] S. Zhao, W. Xu, W. Jiang et al., "Regulation of cellular metabolism by protein lysine acetylation," Science, vol. 327, no. 5968, pp. 1000-1004, 2010.

[20] Q. Wang, Y. Zhang, C. Yang et al., "Acetylation of metabolic enzymes coordinates carbon source utilization and metabolic flux," Science, vol. 327, no. 5968, pp. 1004-1007, 2010. 
[21] B. T. Weinert, S. A. Wagner, H. Horn et al., "Proteomewide mapping of the Drosophila acetylome demonstrates a high degree of conservation of lysine acetylation," Science Signaling, vol. 4, no. 183, p. ra48, 2011.

[22] I. Finkemeier, M. Laxa, L. Miguet, A. J. M. Howden, and L. J. Sweetlove, "Proteins of diverse function and subcellular location are lysine acetylated in Arabidopsis," Plant Physiology, vol. 155, no. 4, pp. 1779-1790, 2011.

[23] V. Jeffers and W. J. Sullivan Jr, "Lysine acetylation is widespread on proteins of diverse function and localization in the protozoan parasite toxoplasma gondii," Eukaryotic Cell, vol. 11, no. 6, pp. 735-742, 2012.

[24] G. C. Toro and N. Galanti, "H1 histone and histone variants in Trypanosoma cruzi," Experimental Cell Research, vol. 174, no. 1, pp. 16-24, 1988.

[25] G. C. Toto, C. Wernstedt, C. Medina, N. Jaramillo, U. Hellman, and N. Galanti, "Extremely divergent histone H4 sequence from Trypanosoma cruzi: evolutionary implications," Journal of Cellular Biochemistry, vol. 49, no. 3, pp. 266271, 1992.

[26] H. Hecker, B. Betschart, K. Bender, M. Burri, and W. Schlimme, "The chromatin of trypanosomes," International Journal for Parasitology, vol. 24, no. 6, pp. 809-819, 1994.

[27] W. J. Sullivan Jr, A. Naguleswaran, and S. O. Angel, "Histones and histone modifications in protozoan parasites," Cellular Microbiology, vol. 8, no. 12, pp. 1850-1861, 2006.

[28] J. P. C. da Cunha, E. S. Nakayasu, I. C. de Almeida, and S. Schenkman, "Post-translational modifications of Trypanosoma cruzi histone H4," Molecular and Biochemical Parasitology, vol. 150, no. 2, pp. 268-277, 2006.

[29] C. J. Janzen, J. P. Fernandez, H. Deng, R. Diaz, S. B. Hake, and G. A. M. Cross, "Unusual histone modifications in Trypanosoma brucei," FEBS Letters, vol. 580, no. 9, pp. 2306 2310, 2006.

[30] V. Mandava, C. J. Janzen, and G. A. M. Cross, “Trypanosome $\mathrm{H} 2 \mathrm{Bv}$ replaces $\mathrm{H} 2 \mathrm{~B}$ in nucleosomes enriched for $\mathrm{H} 3 \mathrm{~K} 4$ and K76 trimethylation," Biochemical and Biophysical Research Communications, vol. 368, no. 4, pp. 846-851, 2008.

[31] A. C. Ivens, C. S. Peacock, E. A. Worthey et al., "The genome of the kinetoplastid parasite, Leishmania major," Science, vol. 309, no. 5733, pp. 436-442, 2005.

[32] J. P. Daniels, K. Gull, and B. Wickstead, "Cell biology of the trypanosome genome," Microbiology and Molecular Biology Reviews, vol. 74, no. 4, pp. 552-569, 2010.

[33] S. C. Nardelli, J. P. C. Cunha, M. C. M. Motta, and S. Schenkman, "Distinct acetylation of Trypanosoma cruzi histone $\mathrm{H} 4$ during cell cycle, parasite differentiation, and after DNA damage," Chromosoma, vol. 118, no. 4, pp. 487499, 2009.

[34] T. Kawahara, T. N. Siegel, A. K. Ingram, S. Alsford, G. A. M. Cross, and D. Horn, "Two essential MYST-family proteins display distinct roles in histone H4K10 acetylation and telomeric silencing in trypanosomes," Molecular Microbiology, vol. 69, no. 4, pp. 1054-1068, 2008.

[35] D. Kumar, K. Rajanala, N. Minocha, and S. Saha, "Histone $\mathrm{H} 4$ lysine 14 acetylation in Leishmania donovani is mediated by the MYST-family protein HAT4," Microbiology, vol. 158, part 2, pp. 328-337, 2012.

[36] S. Alsford and D. Horn, "Elongator protein 3b negatively regulates ribosomal DNA transcription in African trypanosomes," Molecular and Cellular Biology, vol. 31, no. 9, pp. 1822-1832, 2011.
[37] T. N. Siegel, D. R. Hekstra, L. E. Kemp et al., "Four histone variants mark the boundaries of polycistronic transcription units in Trypanosoma brucei," Genes and Development, vol. 23, no. 9, pp. 1063-1076, 2009.

[38] S. Thomas, A. Green, N. R. Sturm, D. A. Campbell, and P. J. Myler, "Histone acetylations mark origins of polycistronic transcription in Leishmania major," BMC Genomics, vol. 10, p. 152, 2009.

[39] R. Sterner, G. Vidali, and V. G. Allfrey, "Studies of acetylation and deacetylation in high mobility group proteins. Identification of the sites of acetylation in HMG-1.," Journal of Biological Chemistry, vol. 254, no. 22, pp. 11577-11583, 1979.

[40] M. Morales, E. Onate, M. Imschenetzky, and N. Galanti, "HMG-like chromosomal proteins in Trypanosoma cruzi," Journal of Cellular Biochemistry, vol. 50, no. 3, pp. 279-284, 1992.

[41] P. Cribb, M. Perozzi, G. V. Villanova, A. Trochine, and E. Serra, "Characterization of TcHMGB, a high mobility group B family member protein from Trypanosoma cruzi," International Journal for Parasitology, vol. 41, no. 11, pp. 1149-1156, 2011.

[42] I. Ugrinova, E. Mitkova, C. Moskalenko, I. Pashev, and E. Pasheva, "DNA bending versus DNA end joining activity of HMGB1 protein is modulated in vitro by acetylation," Biochemistry, vol. 46, no. 8, pp. 2111-2117, 2007.

[43] I. Ugrinova, I. G. Pashev, and E. A. Pasheva, "Nucleosome binding properties and co-remodeling activities of native and in vivo acetylated HMGB-1 and HMGB-2 proteins," Biochemistry, vol. 48, no. 27, pp. 6502-6507, 2009.

[44] Q. Zhang and Y. Wang, "High mobility group proteins and their post-translational modifications," Biochimica et Biophysica Acta, vol. 1784, no. 9, pp. 1159-1166, 2008.

[45] R. Assenberg, M. Webb, E. Connolly et al., "A critical role in structure-specific DNA binding for the acetylatable lysine residues in HMGB1," Biochemical Journal, vol. 411, no. 3, pp. 553-561, 2008.

[46] A. K. Ingram and D. Horn, "Histone deacetylases in Trypanosoma brucei: two are essential and another is required for normal cell cycle progression," Molecular Microbiology, vol. 45, no. 1, pp. 89-97, 2002.

[47] Q. P. Wang, T. Kawahara, and D. Horn, "Histone deacetylases play distinct roles in telomeric VSG expression site silencing in African trypanosomes," Molecular Microbiology, vol. 77, no. 5, pp. 1237-1245, 2010.

[48] B. Yahiaoui, A. Taibi, and A. Ouaissi, "A Leishmania major protein with extensive homology to silent information regulator 2 of Saccharomyces cerevisiae," Gene, vol. 169, no. 1, pp. 115-118, 1996.

[49] J. Tavares, A. Ouaissi, N. Santarém et al., "The Leishmania infantum cytosolic SIR2-related protein 1 (LiSIR2RP1) is an $\mathrm{NAD}^{+}$-dependent deacetylase and ADP-ribosyltransferase," Biochemical Journal, vol. 415, no. 3, pp. 377-386, 2008.

[50] J. A. García-Salcedo, P. Gijón, D. P. Nolan, P. Tebabi, and E. Pays, "A chromosomal SIR2 homologue with both histone NAD-dependent ADP-ribosyltransferase and deacetylase activities is involved in DNA repair in Trypanosoma brucei," EMBO Journal, vol. 22, no. 21, pp. 5851-5862, 2003.

[51] S. Alsford, T. Kawahara, C. Isamah, and D. Horn, "A sirtuin in the African trypanosome is involved in both DNA repair and telomeric gene silencing but is not required for antigenic variation," Molecular Microbiology, vol. 63, no. 3, pp. 724736, 2007. 
[52] S. W. L'Hernault and J. L. Rosenbaum, "Chlamydomonas $\alpha$ tubulin is posttranslationally modified in the flagella during flagellar assembly," Journal of Cell Biology, vol. 97, no. 1, pp. 258-263, 1983.

[53] S. W. L'Hernault and J. L. Rosenbaum, "Reversal of the posttranslational modification on Chlamydomonas flagellar $\alpha$-tubulin occurs during flagellar resorption," Journal of Cell Biology, vol. 100, no. 2, pp. 457-462, 1985.

[54] S. W. L'Hernault and J. L. Rosenbaum, "Chlamydomonas $\alpha$ tubulin is posttranslationally modified by acetylation on the $\varepsilon$-amino group of a lysine," Biochemistry, vol. 24 , no. 2, pp. 473-478, 1985.

[55] K. J. Verhey and J. Gaertig, "The tubulin code," Cell Cycle, vol. 6, no. 17, pp. 2152-2160, 2007.

[56] M. A. Cambray-Deakin and R. D. Burgoyne, "Acetylated and detyrosinated alpha-tubulins are co-localized in stable microtubules in rat meningeal fibroblasts.," Cell motility and the cytoskeleton, vol. 8, no. 3, pp. 284-291, 1987.

[57] M. M. Black and P. Keyser, "Acetylation of $\alpha$-tubulin in cultured neurons and the induction of $\alpha$-tubulin acetylation in PC12 cells by treatment with nerve growth factor," Journal of Neuroscience, vol. 7, no. 6, pp. 1833-1842, 1987.

[58] S. Belmadani, C. Poüs, R. Fischmeister, and P. F. Méry, "Posttranslational modifications of tubulin and microtubule stability in adult rat ventricular myocytes and immortalized HL1 cardiomyocytes," Molecular and Cellular Biochemistry, vol. 258, no. 1-2, pp. 35-48, 2004.

[59] C. Janke and M. Kneussel, "Tubulin post-translational modifications: encoding functions on the neuronal microtubule cytoskeleton," Trends in Neurosciences, vol. 33, no. 8, pp. 362372, 2010.

[60] H. D. Chavan, G. Singh, and C. S. Dey, "Confocal microscopic investigation of tubulin distribution and effect of paclitaxel on posttranslationally modified tubulins in sodium arsenite resistant Leishmania donovani," Experimental Parasitology, vol. 116, no. 3, pp. 320-326, 2007.

[61] R. Sasse and K. Gull, "Tubulin post-translational modifications and the construction of microtubular organelles in Trypanosoma brucei.," Journal of Cell Science, vol. 90, p. 4, 1988.

[62] T. Souto-Padron, N. L. Cunha e Silva, and W. de Souza, "Acetylated alpha-tubulin in Trypanosoma cruzi: immunocytochemical localization.," Memorias do Instituto Oswaldo Cruz, vol. 88, no. 4, pp. 517-528, 1993.

[63] A. Schneider, T. Sherwin, and R. Sasse, "Subpellicular and flagellar microtubules of Trypanosoma brucei brucei contain the same $\alpha$-tubulin isoforms," Journal of Cell Biology, vol. 104, no. 3, pp. 431-438, 1987.

[64] P. Close, C. Creppe, M. Gillard et al., "The emerging role of lysine acetylation of non-nuclear proteins," Cellular and Molecular Life Sciences, vol. 67, no. 8, pp. 1255-1264, 2010.

[65] S. Spange, T. Wagner, T. Heinzel, and O. H. Krämer, "Acetylation of non-histone proteins modulates cellular signalling at multiple levels," International Journal of Biochemistry and Cell Biology, vol. 41, no. 1, pp. 185-198, 2009.

[66] C. Dhalluin, J. E. Carlson, L. Zeng, C. He, A. K. Aggarwal, and M. M. Zhou, "Structure and ligand of a histone acetyltransferase bromodomain," Nature, vol. 399, no. 6735, pp. 491-496, 1999.

[67] G. V. Villanova, S. C. Nardelli, P. Cribb et al., “Trypanosoma cruzi bromodomain factor 2 (BDF2) binds to acetylated histones and is accumulated after UV irradiation," International Journal for Parasitology, vol. 39, no. 6, pp. 665-673, 2009.
[68] S. Mujtaba, L. Zeng, and M. M. Zhou, "Structure and acetyllysine recognition of the bromodomain," Oncogene, vol. 26, no. 37, pp. 5521-5527, 2007.

[69] S. Y. Roth, J. M. Denu, and C. D. Allis, "Histone acetyltransferases," Annual Review of Biochemistry, vol. 70, pp. 81-120, 2001.

[70] J. E. Brownell and C. D. Allis, "Special HATs for special occasions: linking histone acetylation to chromatin assembly and gene activation," Current Opinion in Genetics and Development, vol. 6, no. 2, pp. 176-184, 1996.

[71] V. Sapountzi and J. Côté, "MYST-family histone acetyltransferases: beyond chromatin," Cellular and Molecular Life Sciences, vol. 68, no. 7, pp. 1147-1156, 2011.

[72] A. Akhtar and P. B. Becker, "The histone H4 acetyltransferase MOF uses a C2HC zinc finger for substrate recognition," EMBO Reports, vol. 2, no. 2, pp. 113-118, 2001.

[73] T. N. Siegel, T. Kawahara, J. A. DeGrasse, C. J. Janzen, D. Horn, and G. A. M. Cross, "Acetylation of histone H4K4 is cell cycle regulated and mediated by HAT3 in Trypanosoma brucei," Molecular Microbiology, vol. 67, no. 4, pp. 762-771, 2008.

[74] R. Marmorstein and S. Y. Roth, "Histone acetyltransferases: function, structure, and catalysis," Current Opinion in Genetics and Development, vol. 11, no. 2, pp. 155-161, 2001.

[75] C. Creppe and M. Buschbeck, "Elongator: an ancestral complex driving transcription and migration through protein acetylation," Journal of Biomedicine and Biotechnology, vol. 2011, Article ID 924898, 8 pages, 2011.

[76] J. S. Akella, D. Wloga, J. Kim et al., "MEC-17 is an $\alpha$-tubulin acetyltransferase," Nature, vol. 467, no. 7312, pp. 218-222, 2010.

[77] X. J. Yang and E. Seto, "The Rpd3/Hda1 family of lysine deacetylases: from bacteria and yeast to mice and men," Nature Reviews Molecular Cell Biology, vol. 9, no. 3, pp. 206218, 2008.

[78] C. B. Brachmann, J. M. Sherman, S. E. Devine, E. E. Cameron, L. Pillus, and J. D. Boeke, "The SIR2 gene family, conserved from bacteria to humans, functions in silencing, cell cycle progression, and chromosome stability," Genes and Development, vol. 9, no. 23, pp. 2888-2902, 1995.

[79] C. Hubbert, A. Guardiola, R. Shao et al., "HDAC6 is a microtubule-associated deacetylase," Nature, vol. 417, no. 6887, pp. 455-458, 2002.

[80] M. C. Haigis and D. A. Sinclair, "Mammalian sirtuins: biological insights and disease relevance," Annual Review of Pathology, vol. 5, pp. 253-295, 2010.

[81] K. G. Tanner, J. Landry, R. Sternglanz, and J. M. Denu, "Silent information regulator 2 family of NAD-dependent histone/protein deacetylases generates a unique product, 1O-acetyl-ADP-ribose," Proceedings of the National Academy of Sciences of the United States of America, vol. 97, no. 26, pp. 14178-14182, 2000.

[82] E. Michishita, J. Y. Park, J. M. Burneskis, J. C. Barrett, and I. Horikawa, "Evolutionarily conserved and nonconserved cellular localizations and functions of human SIRT proteins," Molecular Biology of the Cell, vol. 16, no. 10, pp. 4623-4635, 2005.

[83] B. J. North, B. L. Marshall, M. T. Borra, J. M. Denu, and E. Verdin, "The human Sir2 ortholog, SIRT2, is an $\mathrm{NAD}^{+}$dependent tubulin deacetylase," Molecular Cell, vol. 11, no. 2, pp. 437-444, 2003.

[84] S. C. Dryden, F. A. Nahhas, J. E. Nowak, A. S. Goustin, and M. A. Tainsky, "Role for human SIRT2 NAD-dependent 
deacetylase activity in control of mitotic exit in the cell cycle," Molecular and Cellular Biology, vol. 23, no. 9, pp. 3173-3185, 2003.

[85] M. R. Fessel, C. B. Lira, S. Giorgio, C. H. Ramos, and M. I. Cano, "Sir2-related protein 1 from Leishmania amazonensis is a glycosylated $\mathrm{NAD}^{+}$-dependent deacetylase," Parasitology, vol. 138, no. 10, pp. 1245-1258, 2011.

[86] R. A. Frye, "Characterization of five human cDNAs with homology to the yeast SIR2 gene: Sir2-like proteins (Sirtuins) metabolize NAD and may have protein ADP-ribosyltransferase activity," Biochemical and Biophysical Research Communications, vol. 260, no. 1, pp. 273-279, 1999.

[87] M. Dickinson, R. W. Johnstone, and H. M. Prince, "Histone deacetylase inhibitors: potential targets responsible for their anti-cancer effect," Investigational New Drugs, vol. 28, supplement 1, pp. S3-S20, 2010.

[88] M. Göttlicher, S. Minucci, P. Zhu et al., "Valproic acid defines a novel class of HDAC inhibitors inducing differentiation of transformed cells," EMBO Journal, vol. 20, no. 24, pp. 69696978, 2002.

[89] M. G. Riggs, R. G. Whittaker, J. R. Neumann, and V. M. Ingram, "n-Butyrate causes histone modification in HeLa and Friend erythroleukaemia cells," Nature, vol. 268, no. 5619, pp. 462-464, 1977.

[90] M. Yoshida, M. Kijima, M. Akita, and T. Beppu, "Potent and specific inhibition of mammalian histone deacetylase both in vivo and in vitro by trichostatin A," Journal of Biological Chemistry, vol. 265, no. 28, pp. 17174-17179, 1990.

[91] A. V. Chavan and R. R. Somani, "HDAC inhibitors-new generation of target specific treatment.," Mini Reviews in Medicinal Chemistry, vol. 10, no. 13, pp. 1263-1276, 2010.

[92] T. Araki, Y. Sasaki, and J. Milbrandt, "Increased nuclear NAD biosynthesis and SIRT1 activation prevent axonal degeneration," Science, vol. 305, no. 5686, pp. 1010-1013, 2004.

[93] A. Bedalov, T. Gatbonton, W. P. Irvine, D. E. Gottschling, and J. A. Simon, "Identification of a small molecule inhibitor of Sir2p," Proceedings of the National Academy of Sciences of the United States of America, vol. 98, no. 26, pp. 15113-15118, 2001.

[94] M. Fulco, R. L. Schiltz, S. Iezzi et al., "Sir2 regulates skeletal muscle differentiation as a potential sensor of the redox state," Molecular Cell, vol. 12, no. 1, pp. 51-62, 2003.

[95] F. Yeung, J. E. Hoberg, C. S. Ramsey et al., "Modulation of NF- $\kappa \mathrm{B}$-dependent transcription and cell survival by the SIRT1 deacetylase," EMBO Journal, vol. 23, no. 12, pp. 23692380, 2004.

[96] K. J. Bitterman, R. M. Anderson, H. Y. Cohen, M. LatorreEsteves, and D. A. Sinclair, "Inhibition of silencing and accelerated aging by nicotinamide, a putative negative regulator of yeast Sir2 and human SIRT1," Journal of Biological Chemistry, vol. 277, no. 47, pp. 45099-45107, 2002.

[97] M. V. W. Rekowski and A. Giannis, "Histone acetylation modulation by small molecules: a chemical approach," Biochimica et Biophysica Acta, vol. 1799, no. 10-12, pp. 760767, 2010.

[98] S. J. Darkin-Rattray, A. M. Gurnett, R. W. Myers et al., "Apicidin: a novel antiprotozoal agent that inhibits parasite histone deacetylase," Proceedings of the National Academy of Sciences of the United States of America, vol. 93, no. 23, pp. 13143-13147, 1996.

[99] P. J. Murray, M. Kranz, M. Ladlow et al., "The synthesis of cyclic tetrapeptoid analogues of the antiprotozoal natural product apicidin," Bioorganic and Medicinal Chemistry Letters, vol. 11, no. 6, pp. 773-776, 2001.

[100] J. S. Strobl, M. Cassell, S. M. Mitchell, C. M. Reilly, and D. S. Lindsay, "Scriptaid and suberoylanilide hydroxamic acid are histone deacetylase inhibitors with potent anti-Toxoplasma gondii activity in vitro," Journal of Parasitology, vol. 93, no. 3 , pp. 694-700, 2007.

[101] J. Tan, S. Cang, Y. Ma, R. L. Petrillo, and D. Liu, "Novel histone deacetylase inhibitors in clinical trials as anti-cancer agents," Journal of Hematology and Oncology, vol. 3, p. 5, 2010.

[102] D. Sereno, A. Monte Alegre, R. Silvestre, B. Vergnes, and A. Ouaissi, "In vitro antileishmanial activity of nicotinamide," Antimicrobial Agents and Chemotherapy, vol. 49, no. 2, pp. 808-812, 2005.

[103] K. T. Andrews, A. Walduck, M. J. Kelso, D. P. Fairlie, A. Saul, and P. G. Parsons, "Anti-malarial effect of histone deacetylation inhibitors and mammalian tumour cytodifferentiating agents," International Journal for Parasitology, vol. 30, no. 6, pp. 761-768, 2000.

[104] B. Vergnes, L. Vanhille, A. Ouaissi, and D. Sereno, "Stagespecific antileishmanial activity of an inhibitor of SIR2 histone deacetylase," Acta Tropica, vol. 94, no. 2, pp. 107-115, 2005.

[105] C. J. Merrick and M. T. Duraisingh, "Plasmodium falciparum Sir2: an unusual sirtuin with dual histone deacetylase and ADP-ribosyltransferase activity," Eukaryotic Cell, vol. 6, no. 11, pp. 2081-2091, 2007.

[106] J. M. Kelly, M. C. Taylor, D. Horn, E. Loza, I. Kalvinsh, and F. Bjorkling, "Inhibitors of human histone deacetylase with potent activity against the African trypanosome Trypanosoma brucei," Bioorganic \& Medicinal Chemistry Letters, vol. 22, no. 5, pp. 1886-1890, 2012.

[107] T. Koide, M. Nose, Y. Ogihara, Y. Yabu, and N. Ohta, "Leishmanicidal effect of curcumin in vitro.," Biological \& Pharmaceutical Bulletin, vol. 25, no. 1, pp. 131-133, 2002.

[108] M. Nose, T. Koide, Y. Ogihara, Y. Yabu, and N. Ohta, "Trypanocidal effects of curcumin in vitro," Biological and Pharmaceutical Bulletin, vol. 21, no. 6, pp. 643-645, 1998.

[109] C. Changtam, H. P. de Koning, H. Ibrahim, M. S. Sajid, M. K. Gould, and A. Suksamrarn, "Curcuminoid analogs with potent activity against Trypanosoma and Leishmania species," European Journal of Medicinal Chemistry, vol. 45, no. 3, pp. 941-956, 2010.

[110] L. Cui, J. Miao, T. Furuya et al., "Histone acetyltransferase inhibitor anacardic acid causes changes in global gene expression during in vitro Plasmodium falciparum development," Eukaryotic Cell, vol. 7, no. 7, pp. 1200-1210, 2008.

[111] B. N. Lenta, C. Vonthron-Sénécheau, B. Weniger et al., "Leishmanicidal and cholinesterase inhibiting activities of phenolic compounds from Allanblackia monticola and Symphonia globulifera," Molecules, vol. 12, no. 8, pp. 1548-1557, 2007.

[112] L. M. Figueiredo, G. A. M. Cross, and C. J. Janzen, "Epigenetic regulation in African trypanosomes: a new kid on the block," Nature Reviews Microbiology, vol. 7, no. 7, pp. 504-513, 2009. 

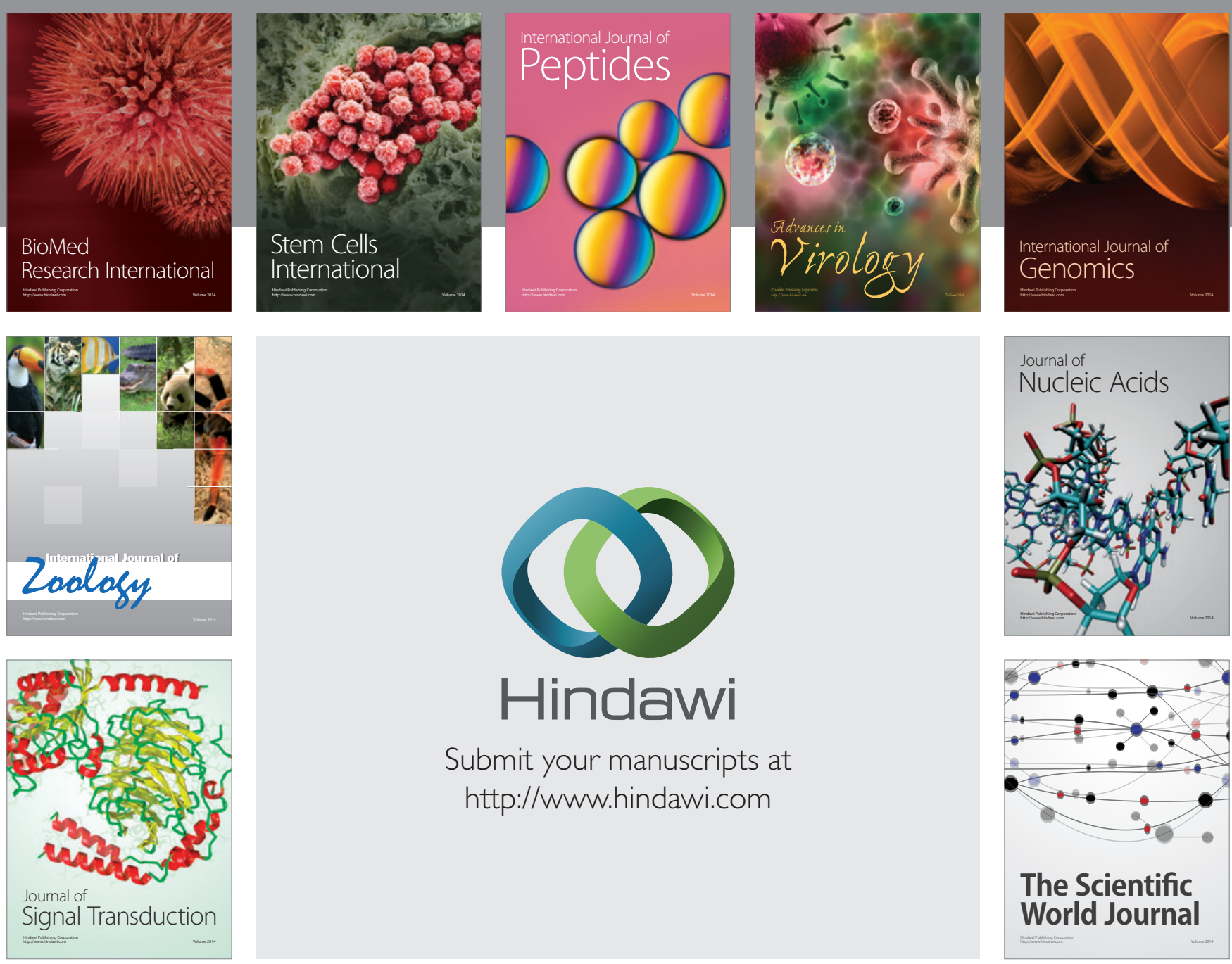

Submit your manuscripts at

http://www.hindawi.com
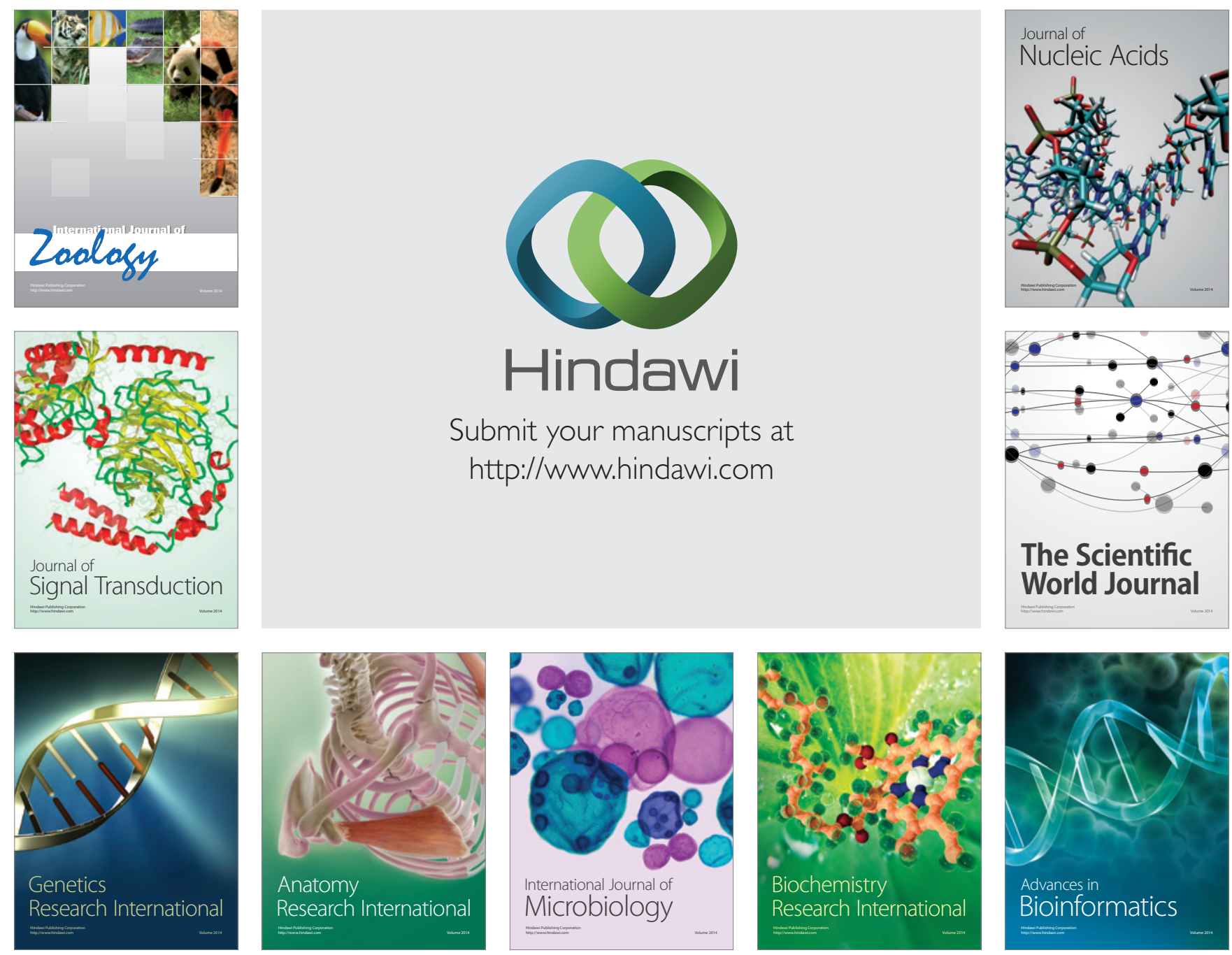

The Scientific World Journal
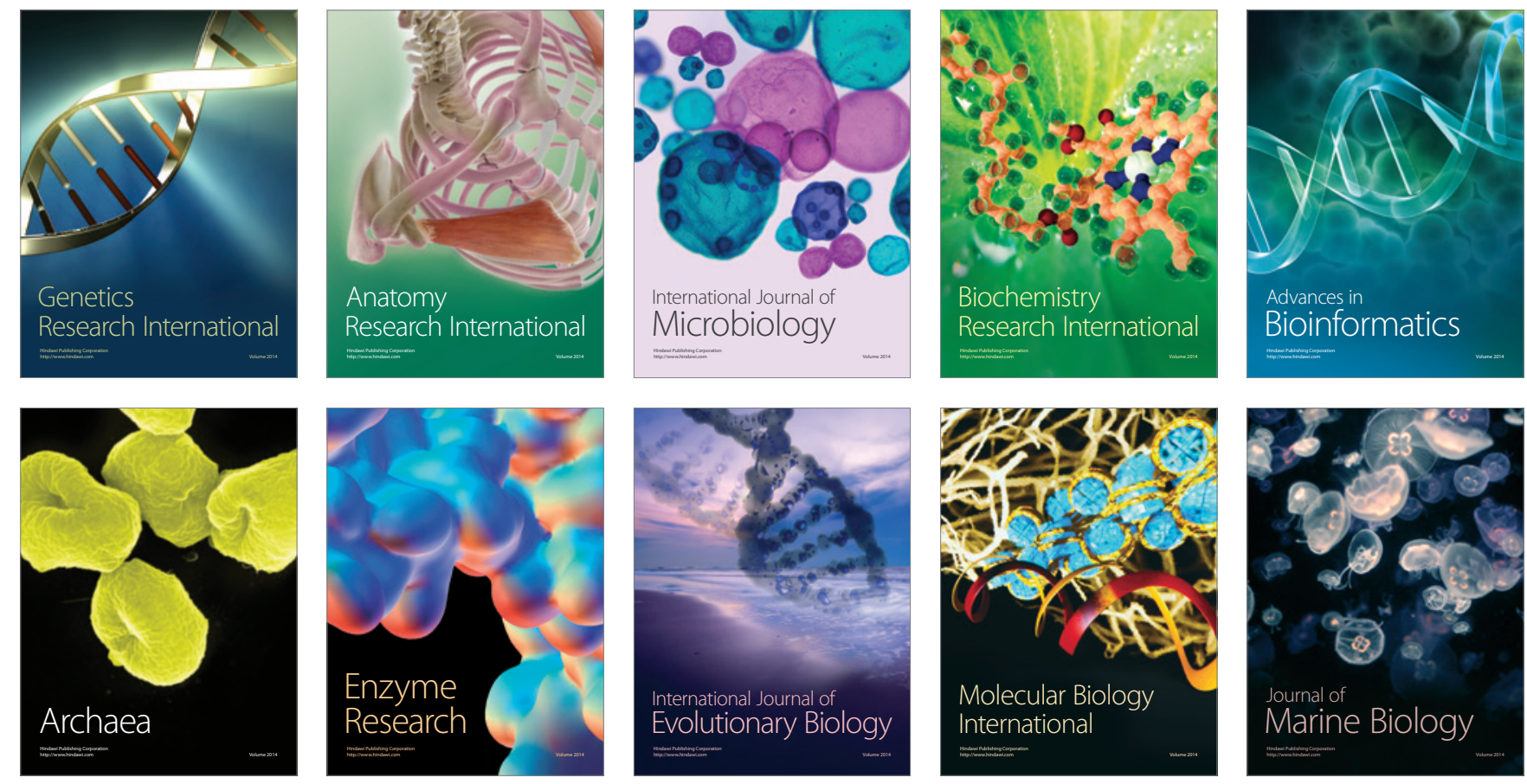